\title{
Emerging systemic delivery strategies of oncolytic viruses: A key step toward cancer immunotherapy
}

\author{
Weiyue Ban ${ }^{1, \S}$, Jianhuan Guan ${ }^{1, \S}$, Hanwei Huang ${ }^{2}$, Zhonggui He${ }^{1}$, Mengchi Sun ${ }^{1}(\varangle)$, Funan Liu ${ }^{2}(\triangle)$, and \\ Jin $\operatorname{Sun}^{1}(\triangle)$ \\ ${ }^{1}$ Wuya College of Innovation, Shenyang Pharmaceutical University, Shenyang 110016, China \\ ${ }^{2}$ Department of Surgical Oncology and General Surgery, The First Hospital of China Medical University; Key Laboratory of Precision Diagnosis and \\ Treatment of Gastrointestinal Tumors, China Medical University, Ministry of Education, Shenyang 110016, China \\ ${ }^{\S}$ Weiyue Ban and Jianhuan Guan contributed equally to this work.
}

(c) Tsinghua University Press and Springer-Verlag GmbH Germany, part of Springer Nature 2021

Received: 28 September 2021 / Revised: 24 November 2021 / Accepted: 28 November 2021

\begin{abstract}
Oncolytic virotherapy (OVT) is a novel type of immunotherapy that induces anti-tumor responses through selective selfreplication within cancer cells and oncolytic virus (OV)-mediated immunostimulation. Notably, talimogene laherparepvec (T-Vec) developed by the Amgen company in 2015, is the first FDA-approved OV product to be administered via intratumoral injection and has been the most successful OVT treatment. However, the systemic administration of OVs still faces huge challenges, including in vivo pre-existing neutralizing antibodies and poor targeting delivery efficacy. Recently, state-of-the-art progress has been made in the development of systemic delivery of OVs, which demonstrates a promising step toward broadening the scope of cancer immunotherapy and improving the clinical efficacy of OV delivery. Herein, this review describes the general characteristics of OVs, focusing on the action mechanisms of OVs as well as the advantages and disadvantages of OVT. The emerging multiple systemic administration approaches of OVs are summarized in the past five years. In addition, the combination treatments between OVT and traditional therapies (chemotherapy, thermotherapy, immunotherapy, and radiotherapy, etc.) are highlighted. Last but not least, the future prospects and challenges of OVT are also discussed, with the aim of facilitating medical researchers to extensively apply the OVT in the cancer therapy.
\end{abstract}

\section{KEYWORDS}

oncolytic virotherapy, oncolytic viruses, talimogene laherparepvec, systemic administration, combination treatments

\section{Introduction}

Currently, people still fear even the mention of cancer. As one of the most fatal diseases, cancer is a great threat to human health [1]. Frequently used anti-tumor therapies in clinical practice include surgical resection, chemotherapy, and radiotherapy (RT), etc. However, multiple biological barriers and low efficiency in the delivery process make it difficult for these methods to work in the tumor microenvironment (TME) $[2,3]$. Considering the fortissimo ability of replication and metastasis of tumor cells, the high probability of cancer recurrence remains an impassable gulf. Therefore, it is imperative to develop novel modalities to treat cancer.

Oncolytic virotherapy (OVT) has become a topic of interest in recent years as a burgeoning immunotherapy. A peculiar occurrence was observed that a tumor-bearing patient unexpectedly went into remission after being infected with the fluenza virus as long ago as 1904. Since OVT was first proposed, several academies and companies have made considerable efforts to improve the clinical applications of oncolytic viruses (OVs). Two OV products are currently being marketed, namely talimogene laherparepvec (T-Vec) (USA) and Ancourt (China) [4]. In addition, dozens of OV products are being tested in clinical trials. Unlocking the potential of OVT has rekindled the hope for cancer patients worldwide.
OVs, involved single/double stranded deoxyribonucleic acid (DNA)/ribonucleic Acid (RNA), are a highly attractive platform for cancer treatment [5]. Although incompletely understood, the antitumor effects of OVs are exerted through three mechanisms of action [6]: specifically infecting tumor cells and replicating in tumor cells, eventually killing them; acting on tumor environment stromal cells to produce neutrophil clumps in the blood vessels, resulting in a collapse in the tumor vascular system, and activating the host's innate and adaptive immune responses against tumors. Furthermore, broken cancer cell fragments are recognized and captured by antigen-presenting cells (APCs), thereby inducing an immune response in $\mathrm{CD}^{+}$and $\mathrm{CD}^{+} \mathrm{T}$-cells [7]. As a result, the combination with other cancer therapies could allow clinicians to make better decisions in clinical applications.

Although OVT has incredible advantages in the treatment of cancer, the clinical practice of commercial OVs is not perfect [8]. First, the administration of OVs remains a challenge that needs to be addressed. The most available OVs for intratumoral administration have already been introduced [9]. Intravenous administration of OVs remains limited due to poor target distribution $[10,11]$, pre-existing specific antiviral immunity, and innate immune response [12]. In addition, the results of clinical investigations have indicated that OVT alone cannot fully satisfy 
the clinical requirements, and the combinational treatment is still essential to enhance therapeutic effectiveness. Systemically administered OVT combined with other therapeutic modalities has been used increasingly in recent years. We have good reason to believe that these promising indications may expand the application scope of OVs.

In this review, we briefly introduce the anti-tumor mechanisms and ongoing clinical trials of OVs, summarize the diverse systemic delivery strategies of OVs in detail, and emphasize the development status of OV-mediated synergistic anti-tumor therapeutic modalities (chemotherapy, thermotherapy, immunotherapy, and radiotherapy). We also discuss the perspectives of OVT in cancer treatments and elucidate the distinct challenges in OV agent development based on both preclinical and clinical data.

\section{Foundation of oncolytic virotherapy}

\subsection{Anti-cancer mechanisms of OVs}

Most of OVs have impressive tumor inhibition capability, however, the exact mechanism remains unclear. Many factors need to be considered, including the type and dosage of virus, the activity of viral replication, and host tumor cell antiviral response. Currently, there are three primary mechanisms have been proposed for common OVs that eliminate cancer cells: directly infecting and killing tumor cells, disrupting the vascular system at the tumor site, and activating anti-tumor immune response.

\subsubsection{The fate of OVs in vivo}

The tumor microenvironment is a complex milieu including a variety of cells including cancer cells, vascular endothelial cells, immune cells, and cancer-associated fibroblasts (CAFs). According to previous report, poliovirus could infect macrophages, convert immunosuppressive cells to a pro-inflammatory phenotype [13]. Furthermore, OVs could interact with neutrophils enriched in TME [14]. OVs infection induces neutrophils repolarization, which would remit the immune suppression though reduction of reactive oxygen species (ROS) and arginase 1 (ARG1) in the TME. In addition, as for regulatory $\mathrm{T}$ cell enriched in TME, OV infection would induce secretion of IFN- $\gamma$, activation of $\mathrm{CD}^{+}$cytotoxic T lymphocytes (CTLs), and a decrease in Treg cell numbers through reduction in transforming growth factor- $\beta$ (TGF $\beta$ ) in the TME. A recent study in B16 bearing mice showed that adenovirus improved the production of tumor-specific T cells by inducing the increase of pro-inflammatory cytokine production. Moreover, OVs therapy would also result in depletion of CAFs. In previous study, TGF- $\beta$ produced by tumor cells reprogrammed CAFs, dampened their steady-state level of antiviral transcripts, and rendered them sensitive to virus infection. CAFs produced high levels of fibroblast growth factor 2 (FGF2), initiating a signaling cascade in cancer cells that reduced retinoic acid-inducible gene I (RIG-I) expression and obstructed the ability of malignant cells to detect and respond to virus. The FGF2-expressed OVs could improve therapeutic efficacy compared to parental virus and require consideration for clinical trial [15]. Additionally, myeloid-derived suppressor cells (MDSCs) could impair OVs efficacy, which prevent the pro-inflammatory response and resultant antitumor immunity [16]. In a recent study, researchers created a recombinant $\mathrm{OV}$ expressing the prostaglandin-inactivating enzyme 15-hydroxyprostaglandin dehydrogenase (HPGD). This could lead to the selective depletion of MDSCs in a mouse kidney cancer model. Therefore, in-depth analysis of the relationships between cells in TME and OVs is helpful for guiding rational OVs selection and combination strategies.

\subsubsection{Directly infecting and killing tumor cells}

The microcosmic mechanism by which cancer cells may preferentially be infected by OVs is ascribed to the changes in intracellular downstream signaling cascades of specific proteins such as RAS, TP53, RB1, and PTEN [17]. Tumorigenesis is a multiple process, including proliferation, replication, apoptosis, angiogenesis, invasion, and metastasis. It depends on activation of oncogenes and inactivation of tumor suppressor genes. Activation of epidermal growth factor receptor (EGFR) leads to upregulation of vascular endothelial growth factor (VEGF) that results in sustained angiogenesis. According to previous report, oncolytic vaccina and vesicular stomatitis virus (VSV) could selectively infect tumor cells and subsequently destroy tumor-associated endothelial in the presence of EGFR due to the upregulation of ras/mitogen-activated protein kinase (MAPK) [18]. In addition, P53 is the most commonly mutated gene in human cancers. Loss of P53 in tumor cells could lead to insensitivity of anti-growth signals. Moreover, activation of Wnt and stabilization of $\beta$-catenin could result in cancer invasion and metastasis. The gammaherpes viruses, such as Epstein-Barr (EBV) and Kaposi's sarcomaassociated herpesvirus (KSHV), have been shown to target the Wnt signaling pathway components. Unlike normal cells, loss of IFN signaling promotes tumor growth and immune evasion. Antagonism of IFN signaling pathway components, including interferon receptor alpha1 (IFNa), INFRA1, IRF1, IRF7, RIG-I, STAT1, and JAK2, could attenuate host antiviral responses. Modified rhabdoviruses, such as VSV and Maraba virus, are prototypical OVs that rely on defects in interferon signaling for selectively infecting tumor cells [19]. As a result, signaling pathways in tumor cells contribute to immortality, unbridled cellular growth, immune suppression, deregulated metabolism. Anormal signaling pathways seem to be incompatible with effective antiviral responses. Thus, tumor cells are more susceptible for viral infection than normal cells [20]. Based on these facilitating conditions offered by cancer cells, natural types (e.g., reovirus) or engineered forms (e.g., herpes simplex virus (HSV)) of viruses can selectively recognize and infect these deviant cells. OVs replication by attaching themselves to host cancer cells and co-opting the host resources to replicate themselves, eventually cleaving the cell. After that, the progeny viruses released from the ruptured cells re-infect the surrounding tumor cells and repeat the process to achieve a macrocosmic tumor-killing landscape. Since numerous previous reviews concerning the replication mechanisms of OVs in cancer cells have been reported, we here did not discuss this phase in detail $[8,21]$.

\subsubsection{Disrupting the vascular system at the tumor site}

OVs, such as adenovirus, vaccinia virus (VV), and measles virus, can also infect and kill endothelial and stromal cells in the TME, both of which play key roles in the tumor development and metastasis. One example is the potential ability of the vesicular stomatitis virus to block angiogenesis, which directly cuts off the tumor oxygen and nutrient supply, decreases inflammation in the TME, and inhibits local microthrombi. Lee et al. reported that BPH-1 cells, a human hyperplastic prostatic epithelial cell line, reduced the expression of angiogenin (a protein that may efficiently stimulate angiogenesis) when these cells received signals from OVs [22]. In addition, engineered OVs may over-express relevant agents to suppress neovasculature formation at tumor sites. For example, an engineered adenovirus can secrete a soluble type of macrophage colony-stimulating factor-1 (CSF1) receptor (CD115) into circulating endothelial progenitor (CEP) cells. Moreover, CD115-expressing CEP cells could target the growth regions of prostate tumors to inhibit neovascularization after intracardial injection [23]. 


\subsubsection{Activating anti-tumor immune response}

Oncolytic viruses are a versatile platform for cancer treatment, and their antitumor immune activity is mediated through multiple mechanisms, including induction of immunogenic cell death (ICD), innate imunity, and adaptive immunity. ICD and endoplasmic reticulum (ER) stress were induced by OVs and eventually led to apoptosis and release of viral pathogen-associated molecular patterns (PAMPs), tumor associated antigen, and damage-associated molecular patterns (DAMPs). The release of these molecules would provide favorable conditions for the activation of innate immunity. PAMPs and virus elements, such as DNA and RNA, could bind to Toll-like receptors (TLRs) and other innate receptors, either on the surface or in the cytosol of DCs, then induce DCs maturation, release cytokines (such as IL-12) and migrate to local draining lymph nodes [24]. In addition, DAMPs could also induce maturation of DCs. For example, extracellular ATP is a chemoattractant for immune cells and plays a key role in the activation of DCs [25]. HMGB1 and calreticulin are ligands for TLR4 on DCs, resulting in the activation of DCs. Furthermore, the detection of viral elements could promote the maturation of DCs by production of pro-inflammatory cytokines, including IL-1 $\beta$, IL-6, IL-12, and tumor necrosis factor (TNF). Moreover, chemokines, including IL-8 and monocyte chemotactic protein 1 (MCP1; also known as CCL2) could also cooperate to recruit and activate innate lymphoid cells. Innate immunity can be augmented by the increased expression of MHC class I, MHC class II, and co-stimulatory molecules, such as CD40, CD80, CD83, and CD86 on DCs, which provides a bridge from innate to adaptive immunity. DCs present processed antigens in a complex with MHC class II and MHC class I molecules to $\mathrm{CD} 4^{+} \mathrm{T}$ cells and $\mathrm{CD}^{+} \mathrm{T}$ cells, respectively. Antigen presentation results in the priming and activation of antigen-specific effector $\mathrm{T}$ cell responses. Activated $\mathrm{T}$ cells proliferate in the tumor-draining lymph node and migrate to the tumor in response to chemokines, such as CXCL9 and CXCL10. The CD8 ${ }^{+} \mathrm{T}$ cells would target to the MHC class I-expressed tumor cells [20]. The adaptive immune response possesses another important trait besides specificity, namely memorability, which is essential to the permanent organism defense process even though it takes more time for an adaptive immune response to build up $[26,27]$. Therefore, a distinct immunological landscape at the tumor tissue is generated owing to OV infection. It would be preferable if OVs injected into the host body activated immune responses effectively and immediately infiltrated tumor tissue. To achieve an ideal therapeutic effect, a weak immune response must be induced using OVs to provide sufficient time for OVs to infect, replicate, and kill tumor cells (Fig. 1).

\subsubsection{Expression of therapeutic genes}

In addition to the aforementioned anti-tumor mechanisms based on the functional characteristics of the OVs themselves, genetic modification of OVs serves as a possible mechanism to treat cancer. Cytokines, small polypeptides, and glycoproteins secreted by immune cells all play important roles in the immune response and anti-tumor mechanism of OVs. Granulocyte-macrophage colony-stimulating factor (GM-CSF) is a functional cytokine capable of recruiting APCs and making them mature, which may improve the therapeutic effect of OVT [28]. Engineered OVs that secrete GM-CSF after parasitizing tumor cells have been developed for cancer treatment. Following the injection of the OV agent, the GM-CSF released from OVs recruits DCs to the infected tumor regions. The DCs then deliver signals from tumor antigens and promote the function of anti-tumor specific T-cells. Similarly, OVs modified by IL-2, IL-12, TNF, or other cytokine genes amplify the number of lymphocytes at the tumor site and enhance the practical therapeutic effect in tumor-bearing mouse models $[29,30]$. In addition, OVs can be designed to release $\mathrm{T}$ cell co-stimulatory molecules that improve $\mathrm{T}$ cell activity. Analogously, designing engineered OVs that express T cell checkpoint molecules to prevent $\mathrm{T}$ cell suppression is also an efficient method to treat cancer [31]. Researchers have also endowed OVs with the ability to express a specific enzyme that can make an inert prodrug cytotoxic. For instance, engineered OVs were designed to express cytosine deaminase, which is conducive to killing tumor cells by translating the nontoxic prodrug 5-fluorocytosine to the anti-tumor drug 5-fluorouracil [32].

\subsection{Clinical researches of OVT}

With several clinical trials currently in progress, the US Food and

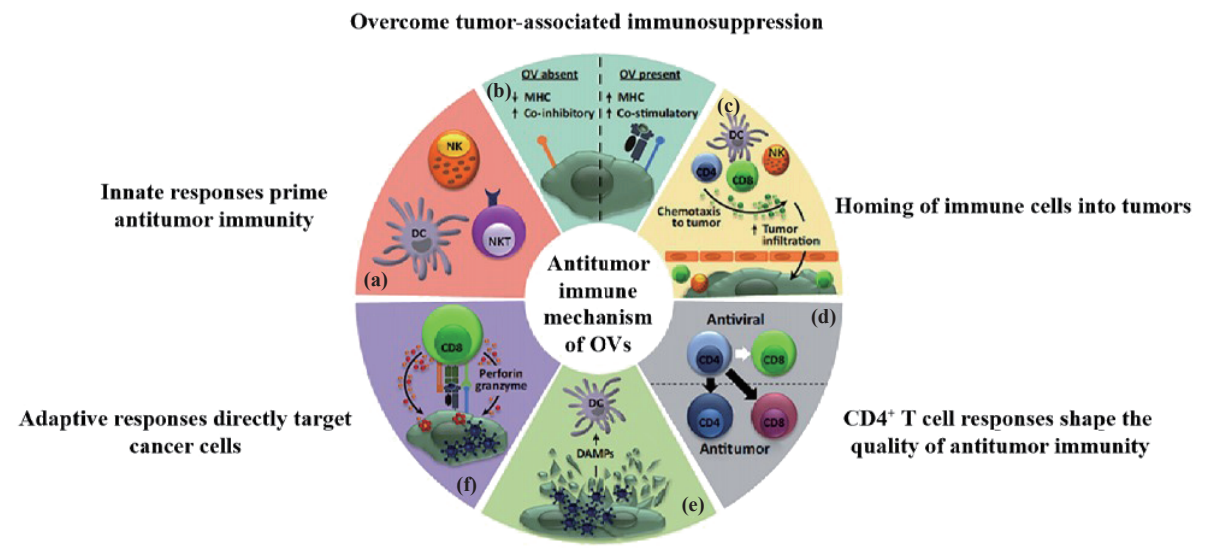

Virus-induced immunogenic cell death activities antitumor immunity

Figure 1 Mechanisms of OVs-mediated antitumour immunity. (a) Functions of virus-activated innate cells like DCs and NK cells are critical for the initial priming of antitumor immune responses. (b) OVs overturn this immune evasion by increasing the expression of MHC, TAP-1/2, and b2M. Similarly, OVs enhance the functions of DCs by increasing co-stimulatory molecule expression on their surface and inducing appropriate cytokines to aid their maturation. (c) Recognition of PAMPs/MAMPs through PRRs promotes the production of multiple pro-inflammatory cytokines that enable homing of immune cells into the tumor microenvironment. (d) CD4 helper T cells aid "licensing" of DCs and can additionally provide "help" for the activation of antitumor CD4 ${ }^{+}$and CD8 ${ }^{+} \mathrm{T}$ cells. In addition, memory antitumor $\mathrm{CD} 4^{+} \mathrm{T}$ cells can affect the functionalities of NK cells. (e) OV-mediated oncolysis of cancer cells bears the typical features of ICD, such as calreticulin surface exposure, HMGB1 and ATP release, ER stress, and increased intracellular $\mathrm{Ca}^{2+}$ levels. DAMPs released through this OV-mediated ICD recruit and activate DCs and lead to the generation of an antitumor immunity. (f) Innate and adaptive responses targeting virus replication sites target cancer cells since oncolytic viruses preferentially infect cancer cells. Reproduced with permission from Ref. [21], (c) Elsevier Ltd. 2017. 
Drug Administration (FDA) proclaimed guidance on the clinical applications of "live biotherapeutic products" with a positive response, which was used to standardize the live biotherapeutic modality. Among the existing live biological antitumor treatments, most clinical studies have focused on OVT, all of which have outlined the fundamental development of OVs in recent years, including common types of $\mathrm{OV}$ and administration, and tumor models. In this review, we gathered information via the "clinical trials" and "PubMed" websites. One hundred and seventeen clinical results came up when "oncolytic virus tumor/cancer" was used as a search term. Adenovirus is the most common OV used clinically in the field of anti-tumor therapy. Almost all the existing clinical studies used intratumor and intravenous administration, and the numbers of both are approximately equal. The common histopathologic types in clinical OVT trials are melanoma and posterior gastrointestinal cancer. The number of clinical studies in phase I $(n=60)$ and phase II $(n=52)$ accounted for $96 \%$ of all studies, and only three were in phase III, as shown in Table 1.

\section{Splendid developing trends of OVs systemic delivery}

Bare or packaged OVs are generally administered via intratumoral injection, which maximally increases $\mathrm{OV}$ distribution in tumor regions [58]. However, this administration method has various flaws [59]: (i) It is difficult to inject OVs smoothly into the tumor due to the compactness and high pressure of the tumor tissue [60]. (ii) In the most cases, intratumoral injection is unsuitable, such as those with malignant tumors in the deep viscera [61]. (iii) Patients who are intratumorally administered have poor compliance, especially those who require continuous administration [8].

Compared with intratumoral injections, intravenous injection seems to be a better approach for the clinical application of OVs $[61,62]$, owing to two distinct advantages: highly improved convenience and feasibility, and excellent anti-metastasis and antirecurrence abilities. Despite these advantages, the practical outcomes of $\mathrm{OV}$ systemic administration are unsatisfactory, in contrast to anticipation. The existing challenges are as follows. First, once the OVs enter the systemic circulation, they take on the role of an alien species that faces several intrinsic obstacles, including non-specific isolation, pre-existing antiviral immunity, and the innate immune response [63]. Subsequently, the concentration retained in the tumor region via systemic administration is lower than that of intratumoral injection after the same dosage of OVs were administered, while the hazard rate of systemic side effects is higher. Systemic injection is no doubt still the most promising method to deliver OVs for cancer treatment once the fatal disadvantages are overcome. In this section, we review scientific research development in the field of OV systemic delivery in recent years.

\subsection{Stem cells-mediated OVs systemic delivery}

Mesenchymal stem cells (MSCs), a type of non-hematopoietic adult multipotent cell isolated from various tissues, have long been implicated to play key roles in the process of cell differentiation and various disease treatments, such as hematological disease, liver cirrhosis, and nervous system diseases [64-66]. MSCs have also

Table 1 Summary of clinical trials of oncolytic virotherapy against cancer in the last three years

\begin{tabular}{|c|c|c|c|c|c|}
\hline Oncolytic virus type & Cancer type & Administration route & Synergistic therapy & Clinical stage & Ref. \\
\hline Herpes simplex virus- 1 (T-Vec) & Melanoma & Intratumor injection & - & Listed agents & [33] \\
\hline Herpes simplex virus- 1 & Pancreatic cancer & Intratumor injection & Chemotherapy & Phase I & {$[34]$} \\
\hline Oncolytic reovirus & Pancreatic cancer & Intravenous injection & Immunotherapy & Phase I b & [35] \\
\hline Oncolytic measles virus & Multiple myeloma & Intravenous injection & - & Phase I & [36] \\
\hline Oncolytic adenovirus ICOVIR-5 & Melanoma & Intravenous injection & - & Phase I & [37] \\
\hline Coxsackievirus A21 (CVA21) & Acute myeloid leukemia & Intravenous injection & Immunotherapy & Phase I & [38] \\
\hline Coxsackievirus A21 & Bladder cancer & Intravesical injection & Immunotherapy & Phase I & [39] \\
\hline Attenuated measles virus (MV) & Solid cancer/leukemia & Intravenous injection & Immunotherapy & Phase I & [40] \\
\hline Oncolytic Vaccinia virus GL-ONC1 & Peritoneal carcinomatosis & Intraperitoneal injection & - & Phase I & [41] \\
\hline Adenovirus VCN-01 & Retinoblastoma & Intravitreous injection & - & Phase I & {$[42]$} \\
\hline Adenovirus DNX-2401 & Malignant glioma & Intratumor injection & - & Phase I & {$[43]$} \\
\hline Oncolytic herpes simplex virus- 1 & Extra-cranial solid cancers & Intratumor injection & - & Phase I & {$[44]$} \\
\hline Oncolytic reovirus & Metastatic colorectal cancer & Intravenous injection & Chemotherapy & Phase II & [45] \\
\hline Oncolytic adenovirus & Epithelial solid tumors & Intravenous injection & - & Phase I & [46] \\
\hline Oncolytic adenovirus & Bladder cancer & Intravesical injection & - & phase II & [47] \\
\hline Oncolytic reovirus & Metastatic colorectal cancer & Intravenous injection & Chemotherapy & Phase I & [48] \\
\hline Reovirus serotype 3 & Non-small cell lung cancer & Intravenous injection & Chemotherapy & phase II & [49] \\
\hline Gammaherpesviruses & HIV-associated lymphomas & Intravenous injection & Immunotherapy & Phase I & {$[50]$} \\
\hline Seneca valley virus (NTX-010) & Extensive-stage SCLC & Intravenous injection & Chemotherapy & Phase II & {$[51]$} \\
\hline Hemagglutinating virus & Malignant melanoma & Intratumor injection & - & phase I/IIa & {$[52]$} \\
\hline Inactivated hemagglutinating virus & Prostate cancer & Intratumor injection & - & Phase I & [53] \\
\hline Serotype 3 reovirus (Pelareorep) & Metastatic breast cancer & Intravenous injection & Chemotherapy & Phase II & {$[54]$} \\
\hline Talimogene Laherparepvec & Melanoma & Intravenous injection & Immunotherapy & Phase II & [55] \\
\hline Oncolytic adenovirus & Diffuse intrinsic pontine glioma & Intratumor injection & Chemotherapy/radiotherapy & Phase I & [56] \\
\hline Talimogene laherparepvec & Melanoma & Intratumor injection & - & Phase IIIb & [57] \\
\hline
\end{tabular}


served as an ideal drug carrier for cancer therapy due to the diversity of source routes, low immunogenicity, and inherent tumor tropism. Reportedly MSCs have been used to encapsulate a variety of cargoes, including small molecule drugs, polypeptide and protein agents, interferons, and interleukins [67, 68].

MSC-encapsulated OVs present a promising systemic administration modality for primary tumor treatment. For example, $\mathrm{Na}$ et al. devised a novel systemic administration model for pancreatic tumor treatment by loading a relaxin (RLX)expressing oncolytic adenovirus (oAd/RLX) into MSCs [69]. As expected, the results of in vitro and in vivo experiments demonstrated that oAd/RLX had been endowed with an eminent tumor tropism and infiltration capabilities by the MSC vector, again indicating the preponderance of MSCs as a cancer treatment. It is worth mentioning that this study has an innovation point. A biodegradable cationic polymer (poly(ethyleneimine)-conjugated poly (CBA-DAH), PCDP) was used to enhance the uptake of OVs into MSCs. MSC-carried systemic delivery models of OVs have been applied in several other primary and secondary cancers alike, such as glioma, ovarian cancer, and hepatoma carcinoma $[69,70]$, all of which prove that MSCs are excellent candidate vectors for the systemic delivery of OVs.

Its exceptional anti-metastasis ability is the most prominent advantage of the systemic delivery, and is the principal factor of OV systemic administration that researchers should explore and develop further. MSCs can protect OVs from neutralizing antibodies (nAbs), immune factors and help target metastatic tumor regions in vivo. A meaningful discovery regarding the driving of conditionally replicating adenoviruses (CRAds) into MSCs for systemic administration to facilitate the treatment of disseminated lung neoplasms induced by metastatic breast cancer (MBC) has been reported. Previously, genetically modified CRAds could resolve the problems encountered during the systemic delivery of OVs to lung metastases in breast carcinoma treatment. A "coxsackie adenovirus receptor (CAR)-independent entry pathway" had been achieved by altering virus fiber proteins to circumvent the high variability and low expression pattern of the primary adenovirus receptor. In addition, a tumor-specific promoter (TSP) was also inserted into CRAds, which could enhance the tumor specificity of CRAds and reduce systemic side effects as much as possible [71]. However, a gap between the practical results and expectations still exists. Inspired by related reports on MSCs, OV formulations for systemic delivery have been reformed by combining engineered CRAds and MSCs. Following a series of screening for cytotoxicity, antineoplastic capability, and tumor tropism, Ad5/3.CXCR4, an engineered Ad5 generated by switching serotype with $\mathrm{Ad} 3$ along with a tumortargeted ligand (CXCR4), was selected for further experiments. Then MSC-wrapped Ad5/3.CXCR4 was prepared, and it was verified that the mean lung weight of a group following systemic administration was comparable to that of a normal control group, and the survival rate of $\mathrm{MBC}$ mice was prolonged.

In addition to $\mathrm{MBC}$, metastatic melanoma (MM) is also a feared diagnosis among patients with tumors because of its strong proliferation and metastatic characteristics. The results of clinical statistical analyses have indicated that more than $50 \%$ of patients with advanced melanoma also develop brain cancer induced by MM [72]. T-Vec, which was the first FDA-approved OV product and was developed by the Amgen company, may activate the antitumor immune response in distant, uninjected cancer regions. However, the overall survival of patients with stage IVM1b and IVM1c who developed metastatic brain tumors induced by MM did not show a significant improvement after T-Vec treatment [73]. Du et al. attempted to reform the treatment approach for MMs of the brain through the intracarotid administration of oncolytic herpes simplex virus (oHSV)-armed MSCs [74]. Using real-time in vivo imaging, Micrometastases and macrometastases of the brain caused by melanoma were observed in patients using real-time in vivo imaging. The MSCs were armed with different oHSV variants, which could effectively target metastatic tumor lesions and significantly improve the survival percentage of tumor-bearing mice (Fig. 2).

Neural stem cells (NSCs), like MSCs, are another widely applied stem cell type in the anti-cancer field. The outstanding intracranial glioma tropism of NSCs has been reported, and many promising agents that use NSCs as carriers against glioma have been designed. The NSC-encapsulated OV delivery system is the most striking among them, and is mainly used in intracranial administration. This administration was not within the scope of this study. Therefore, further NSC-mediated anti-tumor applications of OVs would not be reviewed in this paper.

\subsection{Cell membrane-mediated OVs systemic delivery}

In addition to the above-mentioned stem cells, various biological methods have been used in the systemic administration of OVs, such as extracellular vehicle-wrapped (EVs) OVs and tumor membrane-based hybrid vector systems. These biological materials possess excellent biocompatibility and target delivery properties in vivo, both of which are attributed to the absence of other delivery vehicles. Despite the differences in existing biological hybrid approaches, many similarities in the aspect of design ideas, characteristics of agents, and contents of investigation are introduced here.

\subsubsection{Bioengineered cell membrane nanovehicles}

Extracellular vehicles are a common heterogeneous collection of carriers derived from almost all types of cells. EVs possess multiple advantages that allow them to become ideal carriers, including the capability to prevent cargoes from being identified and eliminated in vivo, overcoming physiological barriers, and biocompatibility and stability. It is worth noting that EVs can easily perform artificial genetic modification, which allows these EV-encapsulated drugs to attain tumor tropism. To date, several types of cargos, including small-molecule drugs, RNA, and vaccine-like antigens, have been wrapped into EVs, all of which have the potential to be a desired delivery system for systemic administration of OVs.

A novel type of bioengineered cell membrane nanovesicle (BCMN) was designed by Peng et al. to systemically deliver OVs for cancer treatment [7]. Researchers have identified a sodium taurocholate co-transporting polypeptide (NTCP) receptor located on the surface of cancer cells that specifically recognizes preS1derived peptide ligands. In this study, two methods of BCMN biomimetic synthesis to achieve tumor tropism are used: embedding relevant ligands on the cell membrane in vitro (abbreviated as in vitro method), and the other uses CRISPRengineered target ligands on erythrocyte-derived BCMNs in vivo $[75,76]$. The results of transmission electron microscopy and dynamic light scattering have indicated that BCMNs can successfully wrap oncolytic adenoviruses (OAs). Using nude mice bearing HepG2-NTCP subcutaneous tumors, OA@BCMNs-preS1 after systemic administration has been shown to possess a long circulation time in vivo and an evident tumor tropism. The HepG2-NTCP orthotopic model was also used to demonstrate that mice maintained a $25 \%$ survival ratio after OA@BCMNspreS1 was administered systemically for 90 days. In contrast, the mice treated with bare OAs died on the $17^{\text {th }}$ day after administration. Likewise, experimental results of OA@BCMNsNGR manufactured in vivo have demonstrated that it is efficient for protecting OVs from nAbs and immune factors and for targeted OV delivery (Fig. 3). 
(a) ICA injection: MeWo-FmC (BRAF Wt) or M12-FmC (BRAF mut)
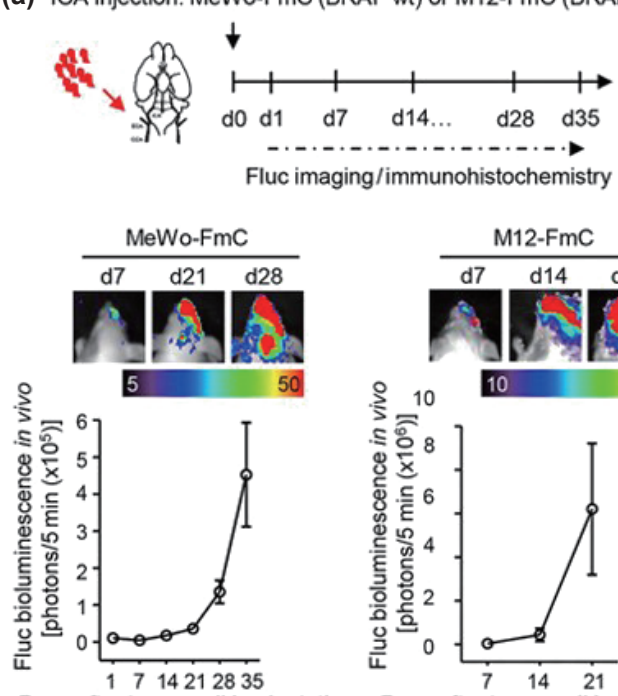

Days after tumor cell implantation

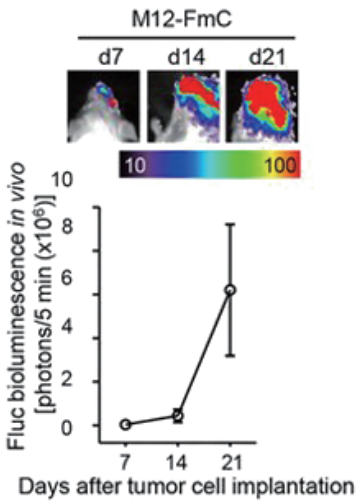

(b)

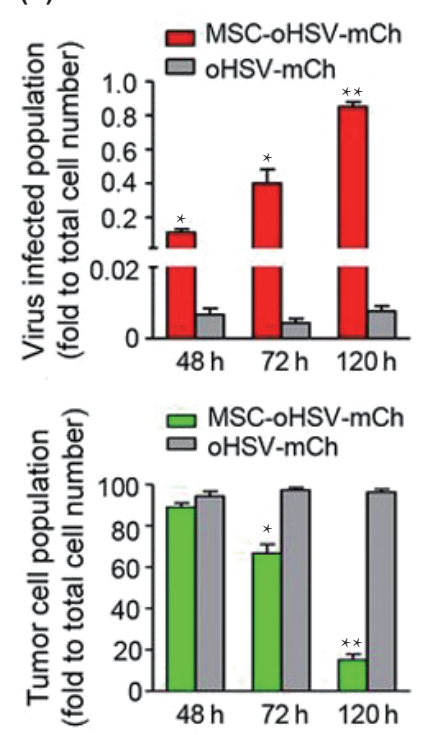

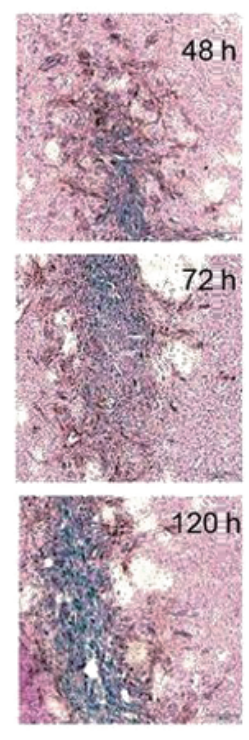
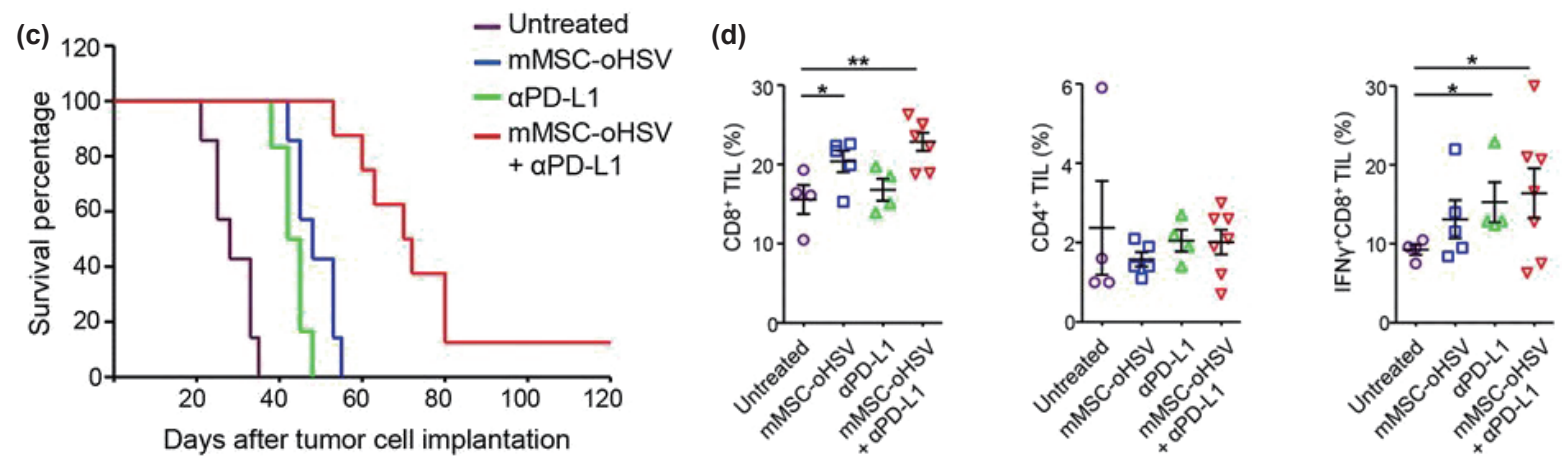

Figure 2 Schematic diagram of oHSV-wrapped by MSC for brain metastatic melanomas treatment. (a) Images illustrating the process of animal model establishment. (b) Diagram illustrating a better tumor infect capability and tumor-kill ability of MSC-oHSV. (c) Survival percentage of model mice at different time after systemic administration. (d) Diagram illustrating the amount of $\mathrm{CD}^{+} \mathrm{T}$ cell, $\mathrm{CD} 4^{+} \mathrm{T}$ cell, and IFN $\gamma^{+} \mathrm{CD}^{+} \mathrm{T}$ cell after systemic administration. Reproduced with permission from Ref. [74], (c) Du, W. et al. 2017.

\subsubsection{Wrapped OVs by cancer cell membrane ExtraCRAd}

Ideally, following systemic OV administration, the tumorassociated antigen would be released and thereby activate $\mathrm{T}$ cells via locoregional immune activation. However, naked OV-induced anti-viral immunity takes precedence over anti-tumor immunity, which results in the quick clearance of OVs in vivo due to a severe host immune response.

Fusciello et al. designed a novel OV nanoparticle (ExtraCRAd) by coating with tumor cell membranes using extrusion technology [77]. Although the ExtraCRAd was injected intratumorally, there are some meaningful features which are worthy of further thinking and learning in this study. On the one hand, one of the defects of Ad is their need for the CAR receptor in order to enter tumor cells and replicate. The infection mechanism of prepared ExtraCRAd is apt to a clathrin-mediated endocytosis effect. Thurs, ExtraCRAd could be considered as an ideal delivery model which can improve infection efficiency of Ad whereas there is no CAR receptors expression. On the other hand, it has been discerned that the shield of tumor cell membranes could protect Ad from recognition and interference of neutralizing antibody in vivo, which paves a promising way for systemic administration of OVs.

\subsection{The serum albumin-mediated OVs systemic delivery}

Luis Alfonso Rojas et al. discovered that binding serum albumin to the surface of adenoviruses is an efficient way to prevent nAbs from destroying the adenovirus [78]. Compared to wrapping the virus in a ready-made cell or membrane structure, this approach would better protect the adenovirus through the aggregation of albumin on the surface of OVs. Specifically, they used genetic modification technology to allow albumin to spontaneously accumulate on the surface of the adenovirus. The results showed that, compared with the original virus (ICOVIR15), although the replication ability and survival time of the modified virus with the albumin-binding domain (ABD) (ICOVIR15-ABD) decreased slightly, its ability to evade nAbs was improved significantly. Based on the aforementioned factors, the circulation time of ICOVIR15$\mathrm{ABD}$ in the body is prolonged, and the actual anti-tumor effect is also improved. Recently, Mato-Berciano et al. have made progress in the field of OVT based on a strategy similar to that mentioned above [79]. In their studies, they used VCN-11, a novel OV, which is genetically modified to express hyaluronidase (PH20) and possesses an $\mathrm{ABD}$ on the hexon, to treat cancer. On the one hand, the expression of $\mathrm{PH} 20$ could favor viral spreading within the tumor, enhance drug delivery, and facilitate immune cell entry into the tumor [80]. On the other hand, the existence of an ABD allows VCN-11 to bind serum albumin and shield itself from nAbs [81]. These results suggested that VCN-11 can induce lower levels of nAbs than that induced by adenovirus-5, facilitating tumor tropism when re-injected, thereby reducing systemic toxicity.

\subsection{Systemic OVs delivery via specifically modifying} key capsid proteins

Neutralizing the OVs with in vivo pre-existing antibodies presents 
(a)

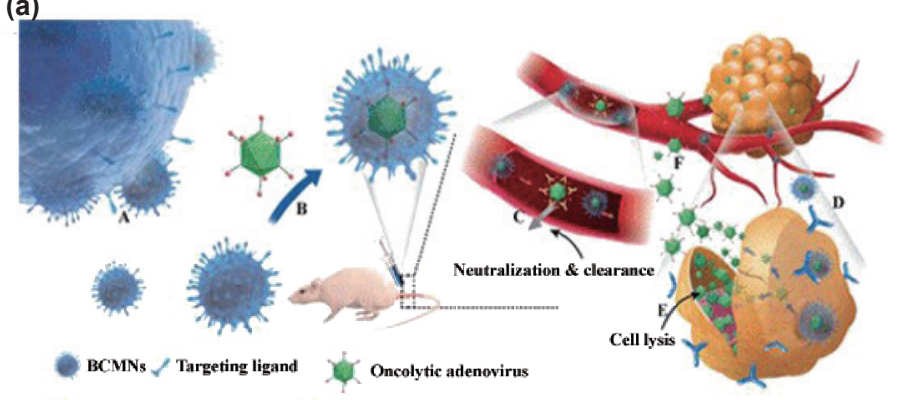

(b)

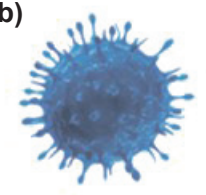

BCMNs

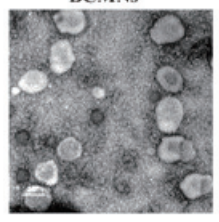

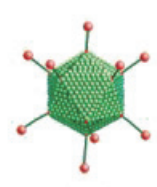

oA

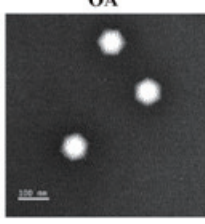

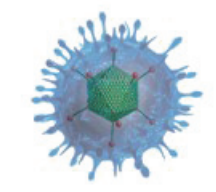

OA@BCMNs

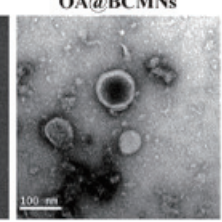

(c)
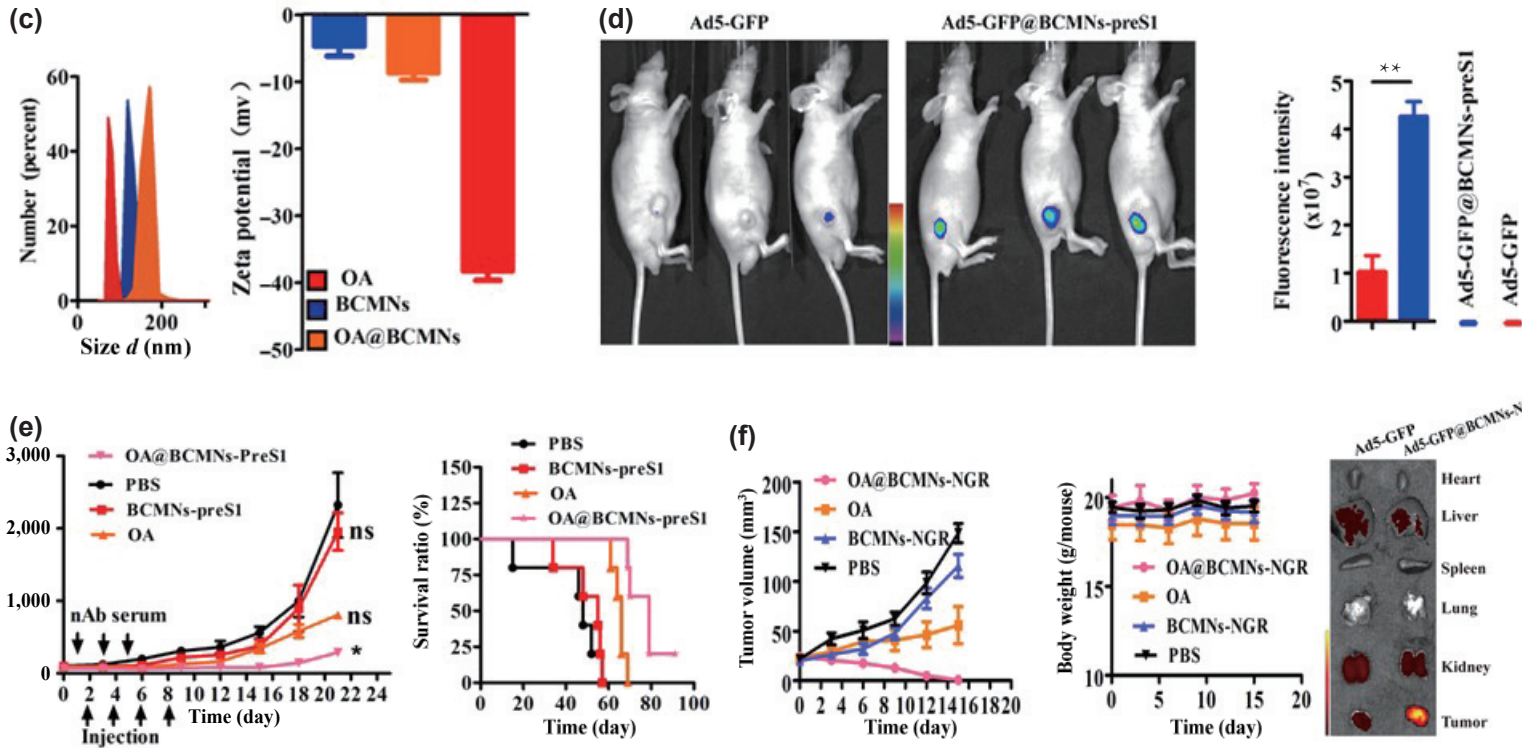

(f)
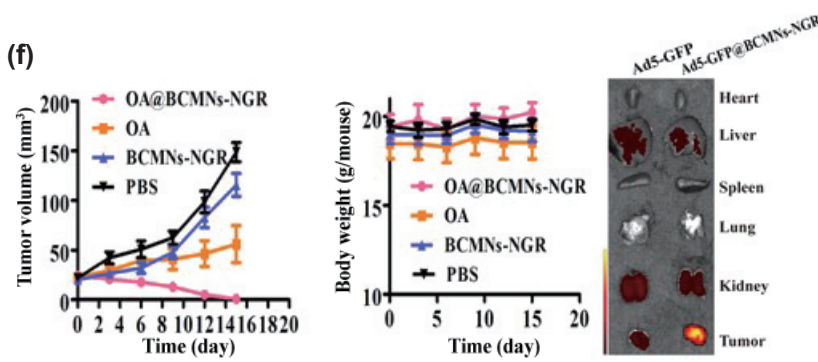

Figure 3 (a) Schematic diagram of Bioengineered cell membrane nanovehicles (BCMNs). (b) TEM images of BCMNs, OA, and OA@BCMNs. (c) Diagram of the size and zeta potential of BCMNs, OA, and OA@BCMNs. (d) Fluorescence images of model mice after 4 days intravenously administration of Ad5-GFP@BCMNspreS1 and Ad5-GFP. (e) Volume of tumor curve and survival ratio chart of model mice after administration. (f) Effect diagram of BCMN manufactured via in vivo method. Reproduced with permission from Ref. [7], ๑ American Chemical Society 2019.

various challenges, as this could result in the invalidation of OVT. The rapid opsonizing effect of antibodies and coagulation factor $\mathrm{X}$ not only impedes the systemic delivery of various OVs, but also causes adverse toxicity in vivo [82-85]. A rare phenomenon was discovered, that is, the fact that the sites of OVs combined with nAbs were fixed in most cases. Taking human species $\mathrm{C}$ adenovirus HAdv-C5 as an example, natural immunoglobulin $\mathrm{M}$ (IgM) antibodies primarily bind to the hypervariable region 1 (HVR1) of the hexon protein, which is a capsid protein on HAdvC5 [78, 86-88]. A novel engineered adenovirus (Ad5-3M) was constructed using specific mutagenesis of virus capsid sites to avoid deactivation during systemic circulation and reduce hepatotoxicity. Specifically, Ad5-3M transforms a mutation (GGSG) in the hexon HVR1 loop to allow Ad5 to escape the combination from Ad-ED2-FX*. A T425A mutation was inserted in the hexon HVR7 loop to avoid the recognition of FX and FXrelated hepatotoxicity and to create a penton-integrin retargeting mutation to prevent the mutual effect of macrophage $\beta_{3}$. Orthotopic disseminated lung tumor-bearing $\mathrm{NCr} /$ nude mice with integrated and fully functional innate immune systems have been used as experimental models to verify the systemic superiority of Ad5-3M. The results showed that Ad5-3M can partially evade the recognition of nAbs during systemic administration. However, not all nAbs lost the ability to recognize AD5-3M (e.g., HAdv-C5-specific nAbs), which may become a limitation of clinical systemic administration. Fortunately, statistical data revealed that $30 \%$ to $70 \%$ of adults in the United States do not possess HAdv-C5-specific nAbs, indicating that there is scope for the development and application of Ad5-3M.

The VSV, which possesses a high degree of cytotoxicity and stability in human serum, is also commonly used in OVT, particularly in the field of systemic administration [89]. However, experimental results showed that VSV concentration decreased by four log units after first exposure to the immune serum [85]. The neutralization velocity was slow compared with that of most OVs, but these results were unsatisfactory and did not allow for future systemic administration of VSV. The Maraba virus has been found to have a better capacity to resist the scavenging of antibodies because of the existence of specific $G$ proteins. Interestingly, the $\mathrm{G}$ protein of Maraba virus was $80 \%$ homologous with that of VSV, which offers the possibility for protein conversion from Maraba virus to VSV. To prolong the cycle time, Tesfay et al. replaced the G gene of VSV with the G gene of Maraba virus and created a novel pseudotyped VSV using genetic engineering technology. Moreover, the results of a series of experiments showed that modified-VSV has a lower seroprevalence than that of parental VSV, but the cell tropism and growth kinetics were extremely similar.

\subsection{The nonbiological vehicle-mediated OVs systemic} delivery

Besides wrapping OVs with biological carrier materials, a variety of non-biological carriers are also used in the field of OVs systemic delivery. Non-biological carrier systems are widespread in the field of cancer treatment previously with various small molecule inhibitors (SMIs) given their superiorities such as biocompatibility, solubility, and extended circulation time compared with free agents [90]. Among them, the representative preparations such as doxorubicin liposome and albumin-bound paclitaxel (PTX) 
nanoparticle both are well known. Notably, OVs possess similar pharmacokinetic and pharmacodynamic properties with SMIs, so it can be judged that non-biological carrier systems of OVs would obtain the same successes and put OVs administration into a brand-new level. In contrast to the biological carrier and gene modification engineering models introduced above, nonbiological carrier systems have many special characteristics. First, there have been many kinds of non-biological carrier materials used in the drug delivery field and their preparation technology and applications are relatively mature. Second, it has high feasibility to design the construct of a systemic delivery model according to the ideas of researchers. Last but not least, it is relatively conducive to the realization of industrialized production. And now some typical applications are listed as follows.

\subsubsection{Biomineralized oncolytic virus}

OA has been regarded as an ideal treatment model and used in the field of cancer treatment, as it possesses specific tumor tropism capability and reliable security. An engineered OA (OA@MnCaCs) carrying OA within calcium and manganese carbonate biomineral shells (MnCaCs) was developed by Huang et al. [91]. This is a sophisticated and efficient nanostructure. They encountered three pertinents during the process of OVT, which were resolved by loading OAs into MnCaCs. First, OA remained undetected by antibodies and could attain long-time blood circulation due to the protection of the MnCaCs shell. In addition,
$\mathrm{OA} @ \mathrm{MnCaCs}$ fully dissolve and release $\mathrm{Mn}^{2+}$, which can reduce ROS to oxygen after its accumulation in the tumor regions. On the one hand, the reduction of ROS benefited the treatment of cancer; on the other hand, the increase in oxygen content could improve the replication ability of OA, thus indirectly enhancing its anti-tumor effect. In addition, it would be feasible to use T1 modal magnetic resonance imaging and photoacoustic imaging to monitor the condition of tumor regions in real-time because of the presence of $\mathrm{Mn}^{2+}$ and $\mathrm{O}_{2}$. Furthermore, after intravenous injection of four relative agents (PBS, OA, OA@ $\mathrm{CaCO}_{3}$, and $\mathrm{OA} @ \mathrm{MnCaCs}$ ) into respective study groups, the in vivo experimental results obtained from BALB/c mice with $4 \mathrm{~T} 1$ xenograft tumors fully demonstrated the anti-cancer capability of $\mathrm{OA} @ \mathrm{MnCaCs}$ in vivo. In summary, the biomineralizaed OV model is efficient for cancer treatment, and it would be wise to further investigate the clinical condition of OA@MnCaCs (Fig. 4).

\subsubsection{Magnetic virus complexes}

Owing to their controllable tropism, magnetic nanoparticles have long been used in the field of drug delivery. Isabella Almstätter et al. attempted to combine magnetic nanoparticle technology with OV delivery to achieve improved systemic delivery of OVs by designing four magnetic virus complex models and conducted a series of experiments to test their anti-cancer capabilities [92]. The results showed that the magnetic virus complex improved the multi-aspect performance of OVs. First, it is convenient that (a)

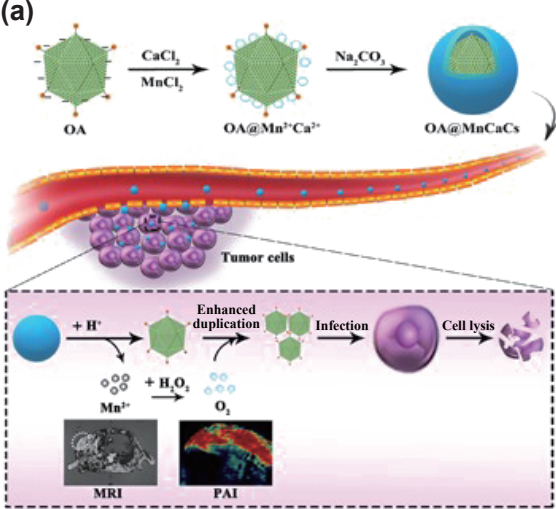

(d) 60
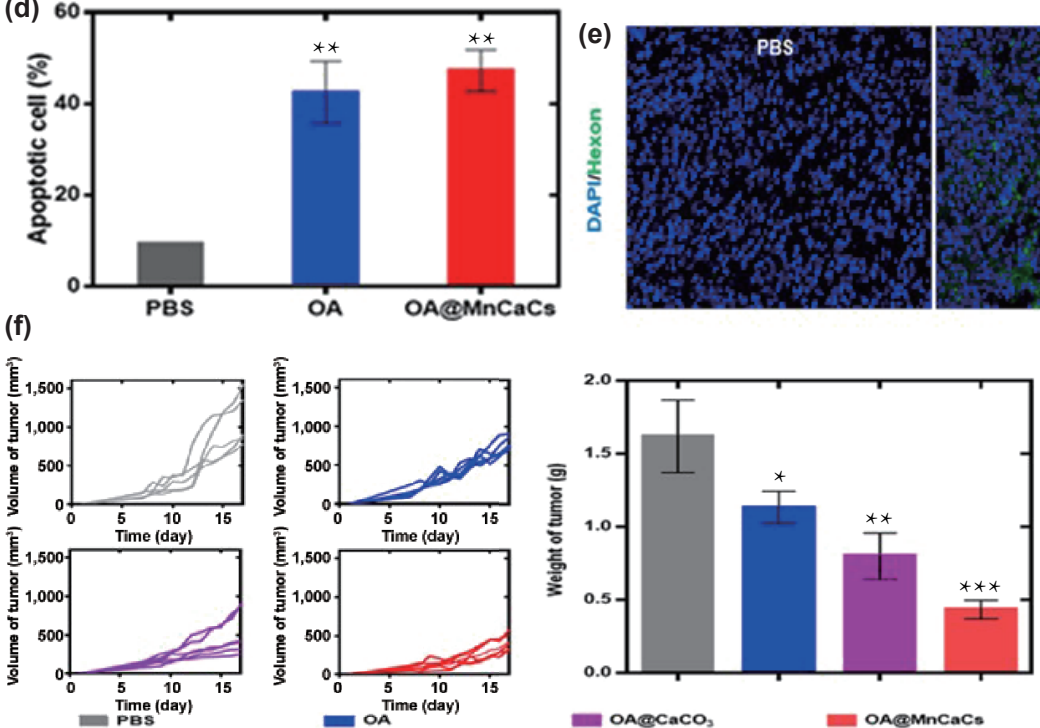

(b)
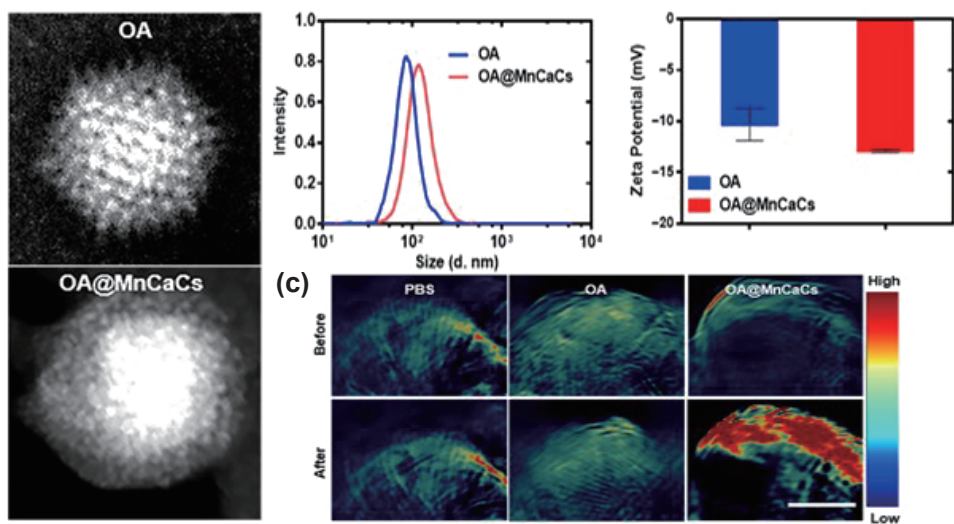

(c)

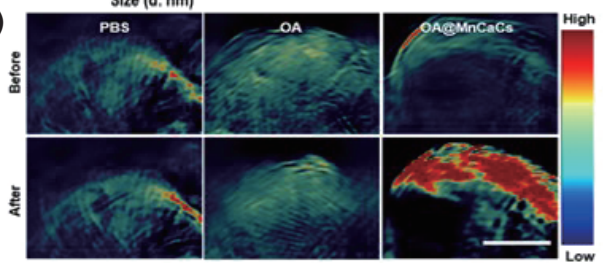

(e)
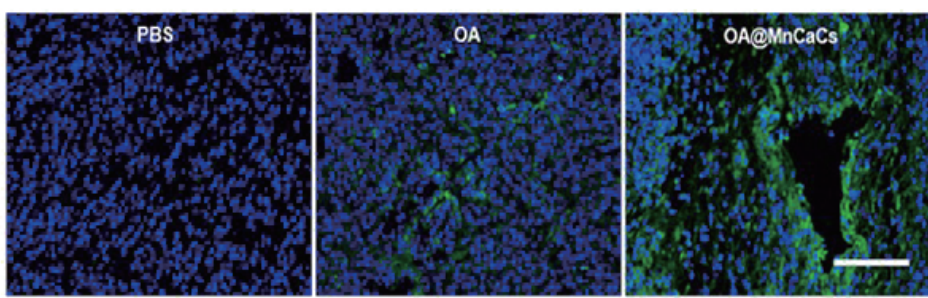

(g)

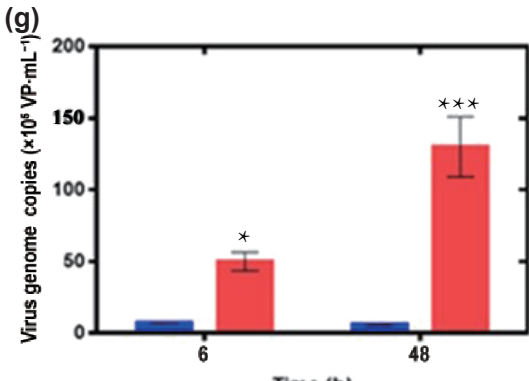

Figure 4 (a) Schematic diagram of biomineralized oncolytic virus (OA@MnCaCs). (b) TEM images, size chart, and zeta potential diagram of OA and OA@MnCaCs (scale bar: $50 \mathrm{~nm}$ ). (c) PA imaging of oxyhemoglobin (HbO2) in 4T1-tumor-bearing mice before and after intravenous administration of PBS, OA, and OA@MnCaCs (scale bar: $3 \mathrm{~mm}$ ). (d) Cytotoxicity of OA and OA@MnCaCs in vitro. (e) Immunofluorescence imaging of tumor sections stained with HIF-1 $\alpha$ (red) and DAPI (blue) (scale bar: $300 \mu \mathrm{m}$ ). (f) Effect diagram of OA@MnCaCs after systemic administration. (g) Using real-time PCR quantifying viral genome copies in tumor after systemic administration 6 and 48 h. Reproduced with permission from Ref. [91], (c) American Chemical Society 2019. 
nanoparticles can be detected in real-time without invasive operations. This also improves its relative safety. Second, due to the action of an external magnetic field, the nanoparticles can effectively target the tumor, thereby improving their efficacy and reducing systemic toxicity. Furthermore, the magnetic nanoparticle shell protects OVs, preventing them from being recognized by $n A b s$ and immune cells in vivo.

In the future, the developments of both OVT and systemic delivery systems will continue to flourish. Therefore, choosing optimal OVs and carriers while understanding the intersection of both would be a notable achievement in the field of OVT. We should not only consider the curative effect of novel agents, but also focus on minimizing toxicity. This is the only way to further propel OVT into the clinical setting.

\subsubsection{Polymer-based OVs nanocarriers}

Owing to excellent biocompatibility and stability, polymer nanocarriers have been used in drug delivery. For example, considering the electrostatic interaction between the positively charged agmatine block and the negatively charged adenoviral capsid surface, Mariangela Garofalo et al. encapsulated OVs into a polygalactosyl-b-agmatyl diblock copolymer (Gal32-b-Agm29) for hepatocellular carcinoma (HCC) remedy, which has high affinity for the asialoglycoprotein receptor (ASGPR) expressed on the liver cell surface [93]. Moreover, Robert Carlisle et al. used cholesterolPEG polymers and anti-MUC1 (aMUC1) antibodies to provide the coating and targeting for an $\mathrm{VV}$ to cellular mucin-1 (MUC1) [94]. Gruanwald et al. evaluated sodium iodide symporter (NIS)-expressing oncolytic Ad coated with polyamidoamine dendrimers G5 (PAMAM G5), which demonstrated enhanced transduction efficacy in CAR-negative cells and protection against neutralizing Abs. Besides, injectable hydrogels are crosslinked polymer networks with large quantity of water and are broadly utilized in biomedical applications. Various attributes of hydrogel serve as an ideal system to address the limitations and enhance the overall potency of oncolytic viruses against cancer. However, hydrogel is more commonly used for topical injection and does not go into details in this review [32].

\section{OVs-mediated cancer combined therapy}

Owing to the existence of tumor heterogeneity, drug resistance, and a variety of innate drawbacks in anti-tumor monotherapies, combined therapies have gradually become mainstream. Satisfactory outcomes have been achieved by combining multiple therapies to treat cancer, such as doublet combination chemotherapy, chemotherapy-radiotherapy, and bacteriamediated synergistic cancer therapy (BMSCT) [95]. Among them, the combination of OVT with other therapies has attracted much attention. In this section, we summarized the development of OVSmediated synergistic cancer therapy. A summary of oncolytic virotherapy combined with other therapies against cancer was shown in Table 2 in clinical and preclinical trials.

\subsection{Synergistic Ovs-mediated chemotherapy}

Chemotherapy remains the most widespread treatment for most cancers, both at early stage and advanced stage. Despite significant recent developments, various gaps still exist in chemotherapybased systems, such as undesirable therapeutic effects and off-target side effects. Given the aforementioned limitations encountered in the treatment process alone, synergistic OVmediated chemotherapies have been proposed to achieve a synergistic effect.

Synergistic OV-mediated chemotherapies have been utilized and approved in China. Oncorine (H101) was approved in 2005 to combine chemotherapeutical drugs to treat advanced

Table 2 Summary of Oncolytic virotherapy combined with other therapies against cancer

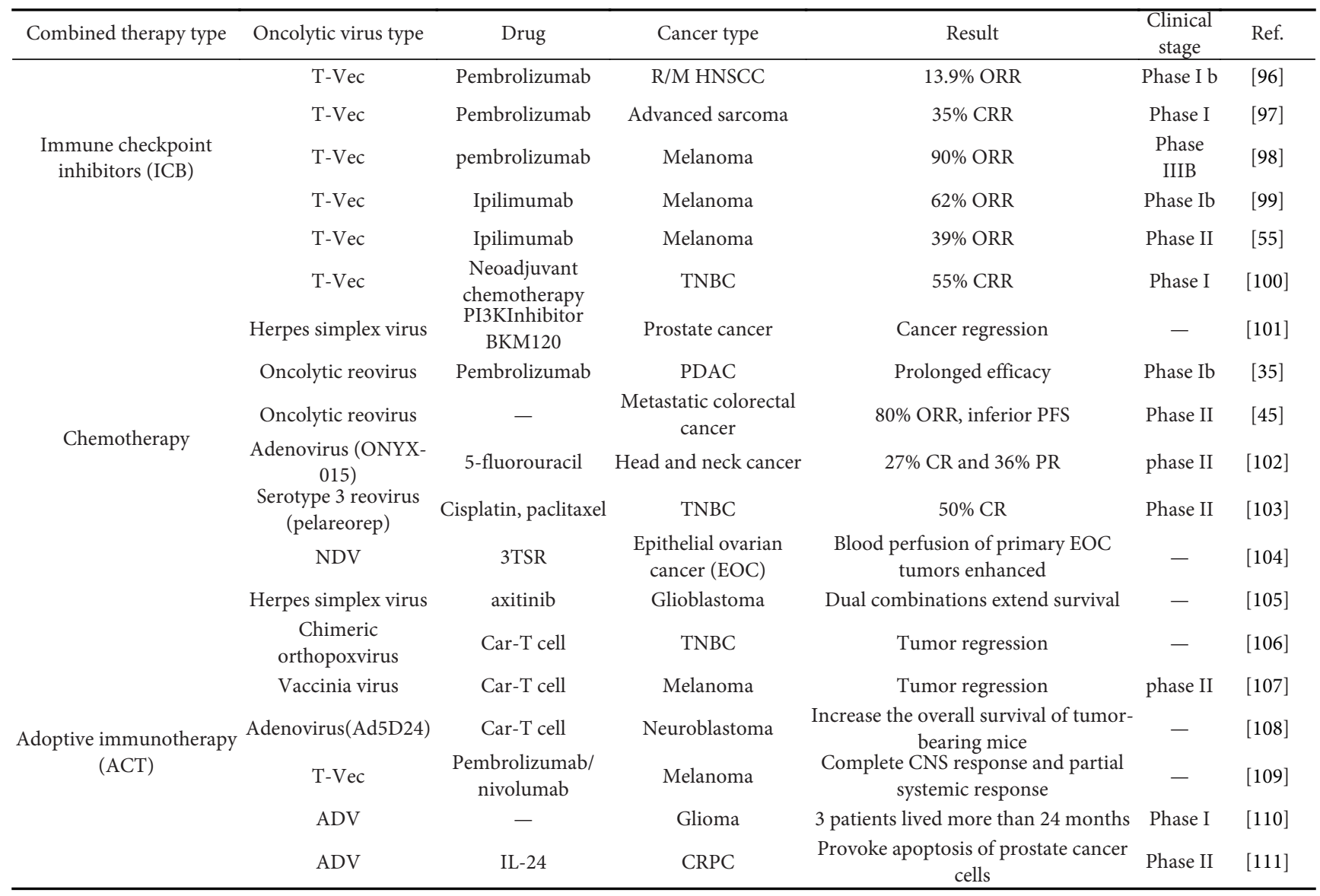


nasopharyngeal cancer. Hitherto, several clinical trials had been conducted, and the concrete mechanisms of synergistic OVmediated chemotherapies were diverse. Some representative instances were as follows: Fadlo R. Khuri et al. have found that ONYX-015, an E18 55Kda gene-deleted adenovirus that possesses the ability to selectively recognize and infect cancer cells with p53-deficient, and had an undesirable therapeutic effect and high recurrence rate when it is used alone to treat recurrent head and neck cancer [102]. However, a satisfactory therapeutic outcome was observed in a phase II trial following combination treatment with cisplatin and 5-fluorouracil. The response rate of the "ONYX-015 alone" group was only 15\%, but that of the combination group was $65 \%$. Thirty cases were evaluated, and the results showed that the tumor volume of 25 patients shrank when they were administered a combination treatment. These outcomes demonstrated that the combination of OVT and chemotherapy could have a synergistic effect against malignant tumors. The mechanism of combining ONYX-015 with cisplatin and 5-fluorouracil suggests that Ovs can more easily enter and kill tumor cells following pretreatment with cisplatin and 5-fluorouracil. In addition, there were existing instances to indicate that stress-induced apoptosis in the ER is an efficient mechanism whereby synergistic OV-mediated chemotherapies function against cancers [112]. Roulstone $\mathrm{V}$ et al. designed a combined method to enhance the anti-tumor ability of reovirus type 3 (RT3D), which is an OV that selectively infects mutant cancer cells with activated RAS-MAPK signal. The rate at which the combination of RT3D with MAPK-ERK kinase inhibitors killed tumor cells was enhanced by stress-induced apoptosis in the ER compared with that of a single RT3D treatment alone. In addition, phase I/II clinical results regarding the use of ONCOS-102 combined with the chemotherapeutic drug SoC to treat malignant pleural mesothelioma (MPM) were announced by Targovax in 2020. The clinical statistics data illustrated that the median progression-free survival of patients who accepted synergistic treatment is longer ( 8.4 months) than that of patients who only received SoC treatment (6.8 months). The overall response rate and disease control rate of the combination therapy group were also significantly improved [113]. Roulstone V et al. designed a combined method to enhance the anti-tumor ability of RT3D, which is an OV that selectively infects mutant cancer cells with activated RAS-MAPK signal. The rate at which the combination of RT3D with MAPK-ERK kinase inhibitors killed tumor cells was enhanced by stress-induced apoptosis in the ER compared with that of a single RT3D treatment alone [100].

\subsection{Synergistic OVs-mediated thermotherapy}

Thermotherapy, also known as "green therapy", has been used to treat various cancers. The therapeutic effect of thermotherapy depends on the difference in tolerance of high temperatures between normal cells and tumor cells. Thermotherapy combats cancer through one of three mechanisms: (1) destroying tumor vascular systems, (2) disrupting tumor cell membranes, and (3) inhibiting the activity of key enzymes for tumor growth and proliferation. However, the therapeutic effect of thermotherapy alone is insufficient, and various complications and side effects are occurred. Thus, the use of thermotherapy alone has not been approved clinically. Due to the abovementioned facts, cases of combining thermotherapy with other modalities are widespread [114]. Among them, synergistic OVS-mediated thermotherapy has attracted attention due to its significant therapeutic effect. For example, to test the feasibility of combining radiofrequency hyperthermia (RFH) with OVT to treat HCC, Song et al. designed the following four in vitro and in vivo trials [115]: (1) T-Vec plus RFH, (2) T-Vec alone, (3) RFH alone, and (4) saline [116]. For in vitro evaluation, McA-RH7777 tumor cells treated with luciferase/red fluorescence protein/lentivirus were used as a trial model. RFH was performed by laying a magnetic resonance imaging heating guidewire under the bottom of the chamber. The minimum survival of cancer cells was observed in the combination therapy group compared with that of the three control groups with confocal microscopy. In addition, quantitative MTS assays have further demonstrated the cancer cell killing ability of the combination of RFH with T-Vec in vitro, and flow cytometry assays have also revealed similar outcomes through estimating apoptosis analysis. In vivo animal experiments have shown that combination therapy has the following therapeutic effects: (1) The relative tumor volume of the combination therapy group was the smallest among the four group outcomes. (2) Optical imaging and histological analysis of apoptosis revealed a reduced relative photon signal in the RFH-TVec combination group. (3) The final specimens obtained via dissection revealed the therapeutic ability of the RFH-T-Vec group based on the visual comparison of tumor sizes.

\subsection{Synergistic OVs-mediated immunotherapy}

In recent years, immunotherapy, a new treatment strategy, has provided the expected improvement in the treatment response in preclinical animal studies [115], and its application in clinical research has also been growing rapidly and has attracted widespread attention. In this section, we focus on two notable synergistic OVs-mediated treatment options: (1) OVs and immune checkpoint inhibitors (ICIs) and (2) OVs combined with CAR-T.

\subsubsection{Combination of OVs and ICIs}

To date, the combination of ICIs with OVs is one of the most promising antitumor approaches against various types of cancer. During tumorigenesis, TMEs may block the immune systemmediated anti-tumor responses through several pathways, including the weakening of tumor antigen presentation, inhibiting negative regulatory factors, activating immune detection point signals to induce $\mathrm{T}$ cell depletion and apoptosis, and competition for nutrients between the TME and immune cells [117, 118]. ICI therapy is clinically applied on the basis of $\mathrm{T}$ cell activation and has been shown to have a breakthrough effect in cancer treatment. The main immune checkpoints include programmed cell death protein 1 (PD-1) and its ligand (PD-L1) or cytotoxic $\mathrm{T}$ lymphocyte-associated protein 4 (CTLA-4) [119]. Anti-CTLA 4 antibodies primarily act at the initial stage of $\mathrm{T}$ cell initiation and activation, while PD1/PD-L1 antibodies primarily act on $\mathrm{T}$ cells that are suppressed. However, many complicated factors may attenuate the ICI treatment effect, such as new tumor antigens associated with cancer mutation load (TMB), the human MHC (the diversity of leukocyte antigen), the expression of tumor cells PD-L1, and infiltrating lymphocytes (TIL) in the absence and inhibition of the TME. PD-1/PD-L1 antibody as a single therapy cannot completely overcome the tumor-specific immune response of immune tolerance-mediated organisms [120]. Therefore, finding an effective combination therapy to activate the immune system and synergistically enhance immune checkpoint blocking therapy is imperative.

The combination of monoclonal antibodies and immune checkpoint molecules (e.g., PD1/PD-L1 and CTLA4-mAb) with OVs has shown clinical efficacy in treating a variety of difficulttreated tumors. Although many patients respond well to checkpoint inhibitors, the management of patients with advanced first-line immunotherapy remains challenging. For example, a patient with diffuse metastatic melanoma, including brain metastases, developed systemic disease progression and numerous 
brain metastases despite stereotactic RT and dual CTLA-4/PD-1 blocking (ipilimumab/nivolumab). This patient achieved a complete central nervous system and partial systemic response with standard whole brain radiation therapy combined with TVec and pembrolizumab [109]. Different combinations of OVs with different ICIs have been applied in clinical research. Given the infiltrating effect of OVs on tumor cells and immune cell activation of ICIs, the combination of OVs and ICIs is used in more than $30 \%$ of the clinical trials with active status. According to a previous study on $\mathrm{T}-\mathrm{Vec}$ combined with pembrolizumab, Ribas et al. found that intratumoral injection of a modified OV to enhance immune recognition produced a high response rate in patients with advanced melanoma [99]. The confirmed objective response rate of $62 \%$ and complete response rate of $33 \%$ for each immune-related response criterion in 21 patients that received this combination of therapy suggested that OVs could enhance the efficacy of anti-PD-1 therapy by altering the TME. The proportion of $\mathrm{CD}^{+} \mathrm{T}$ cells, PD-L1 protein expression, and IFN-G gene expression was increased in several cell subsets in cancer patients who responded to the combination therapy. The above evidence suggested that $\mathrm{OV}$-mediated tumor destruction would change the TME, which would not only promote $\mathrm{T}$ cell infiltration and cause inflammation but also block the immunosuppressive TME through ICI therapy. Moreover, oncolytic immunotherapy has been approved for advanced melanoma in combination with pembrolizumab, demonstrating that the combination of OVs with ICIs could produce stronger antitumor responses than either agent alone in a phase $1 \mathrm{~b}$ study including 36 patients [96]. Sun et al. also reported that 10 consecutive patients with stage IIIC IVM1B melanoma who received a combination of T-Vec and ICIs (pembrolizumab ipilimumab/nivolumab) had improved antitumor response rates [98]. The overall response rate of the target lesion was $90 \%$ in 10 patients, and 6 patients achieved complete response upon injection of the lesion. In addition, it was observed that the $\mathrm{CD} 4 / \mathrm{CD} 8$ radio frequencies were increased in complete responders.

\subsubsection{Combination of $\mathrm{OVs}$ and chimeric antigen receptor (CAR)-T cells}

Adoptive T cell therapies (ACTs) involve the infusion of tumourspecific cytotoxic $\mathrm{T}$ cells into cancer patients. The persistence of ACT is determined by donor T cell transport and survival in the tumour bed [121]. Pretreatment of established tumors with OVs can shape the local tumor microenvironment to improve $\mathrm{T}$ cell recruitment and effector function [1]. Soluble tumor necrosis virus can induce tumor cell necrosis and trigger the release of the new tumor antigens, table a diffusion, and promote new BATF3 ${ }^{+}$DC cells and antigen-specific $\mathrm{CD}^{+} \mathrm{T}$ cell activation, thereby promoting $\mathrm{T}$ cell proliferation. The soluble tumor virus increases MHC class I molecule expression on the surface of cancer cells, which has the potential to enhance ACT through CAR-T cells targeting tumor-specific antigens [8].

Using CAR-T cells to target CD19 has led to achieved longlasting remissions in the treatment of $\mathrm{B}$ cell malignancies, and has also shown remarkable clinical efficacy in the treatment of leukemia and lymphoma [122]. However, the use of CAR-T cells in solid tumors is subject to several limitations. The ability to achieve effective CAR-T extravasation, specific tumor homing, cytotoxic activity, and persistent survival in the TME must be present in these cells. In addition, some solid tumors, including triple-negative breast and liver cancers, lack the tumor antigens required for effective CAR-T cell treatment. In previous studies, OV therapy has been proposed in combination with CAR-T to circumvent the immunosuppressive effects of the TME, reverse $\mathrm{T}$ cell failure, and prevent antigen escape. Armed OVs that express cytokines promoting migration, proliferation, and homing of $\mathrm{T}$ cells to the TME have been successfully combined with CAR-T cell therapies to enhance the antitumor response in tumor models. Herein, we focused on OV and CAR-T combination therapy. OVs can selectively infect and replicate in malignant cells, facilitating the expression of a proverbial target protein called CD19. As the result of CD19 expression occurs much faster than the virus killing the tumor, a window time appears, giving CAR-T the opportunity to target tumors that express the specific CD19 protein. In addition, they have been shown to reprogram the immunosuppressive microenvironment of solid tumors, which could be conducive to $\mathrm{T}$ cell activity enhancement. Moreover, another important part of the anti-tumor mechanism of OVs is that the infected cells cleave and release the OVs, thereby recruiting an endogenous antiviral immune response.

CD19 is a key target for CAR-T cells in the treatment of hematologic malignancies, but it is only expressed on B cells and has undesirable off-targeting properties. Studies using CD19targeted CAR-T cells in large numbers have been approved by the FDA. In a recent report, Park et al. leveraged the transgenic delivery capabilities of OVs to selectively infect and drive the tumor-specific expression of a CAR-targeted tumor antigen CD19 (CD19T) into a non-signaling variant [106]. Studies have shown that (1) CD19T is highly expressed on the surface of multiple OVinfected tumor cells carrying the CD19T-coding gene (OV19T) and subsequently promotes CAR-T cell activation and tumor killing. (2) OV19T promotes the penetration of endogenous $\mathrm{T}$ cells and CAR-T cells into tumors. (3) CD19-CAR T cells can also induce the release of intact OV19T from dead tumor cells. (4) OV19T induces endogenous anti-tumor immunity and promotes tumor invasion by CAR-T cells (Fig. 5).

In a homogenetic mouse model of pancreatic cancer, antimesodermin-CAR-T cells combined with OAs, which simultaneously express tumor necrosis factor $\alpha$ and interleukin-2, significantly promoted tumor eradication. Effective treatment is closely associated with increased accumulation of both CAR-T and host $\mathrm{CD}^{+}$and $\mathrm{CD} 4^{+} \mathrm{T}$ cells within the tumor, and changes in the TME, namely increased macrophage M1 polarization and DC maturation. Further studies were conducted in immunoreactive mouse models to determine the antitumor effect of antiviral immunity on metastatic CAR-T cells and the effectiveness of combination therapy.

The recognized solid tumor antigen targets of CAR-T cells include ganglioside G2 (GD2), mesoderm, B-cell maturation antigen, prostate-specific membrane antigen (PSMA), epidermal growth factor receptor variant III, mucin 1, and esophageal squamous cell carcinoma of New York 1. Further studies on these antigens have the potential to develop future ACTs. Using neuroblastoma as a tumor model, researchers found that adenovirus Ad5D24 exerted a potent, dose-dependent cytotoxic effect on tumor cells, while CAR-T cells exerted a potent, dosedependent cytotoxic effect on GD2 [108]. When in combination with CAR-T, Ad5D24 directly accelerated the caspase pathway in tumor cells exposed to CAR-T cells, while the intratumor release of RANTES and IL-15 attracted CAR-T cells and promoted their local survival, increasing overall survival in tumor-bearing mice. These preclinical data support the use of this innovative combination biological platform for solid tumor immunotherapy.

Rational tumor immunotherapy could include the use of OVs combined with ICI or ACT therapy to induce more immune cells in the TME and produce a sustained anti-tumor immune response. In preclinical tumor models, the combination of OVs with ICIs or ACTs had enhanced or synergistic effects. The development of OVT has changed dramatically, especially after the advent of FDA-approved T-Vec for the treatment of advanced 
(a)

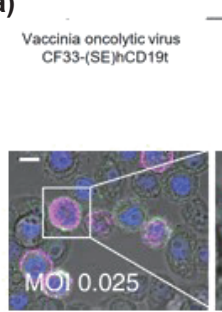

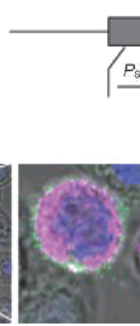

(b)

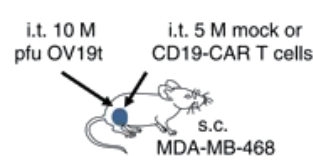

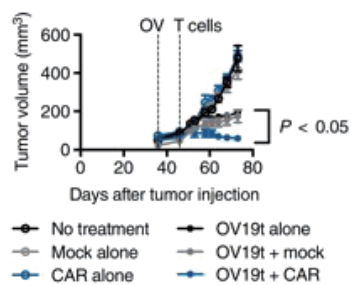

(c)
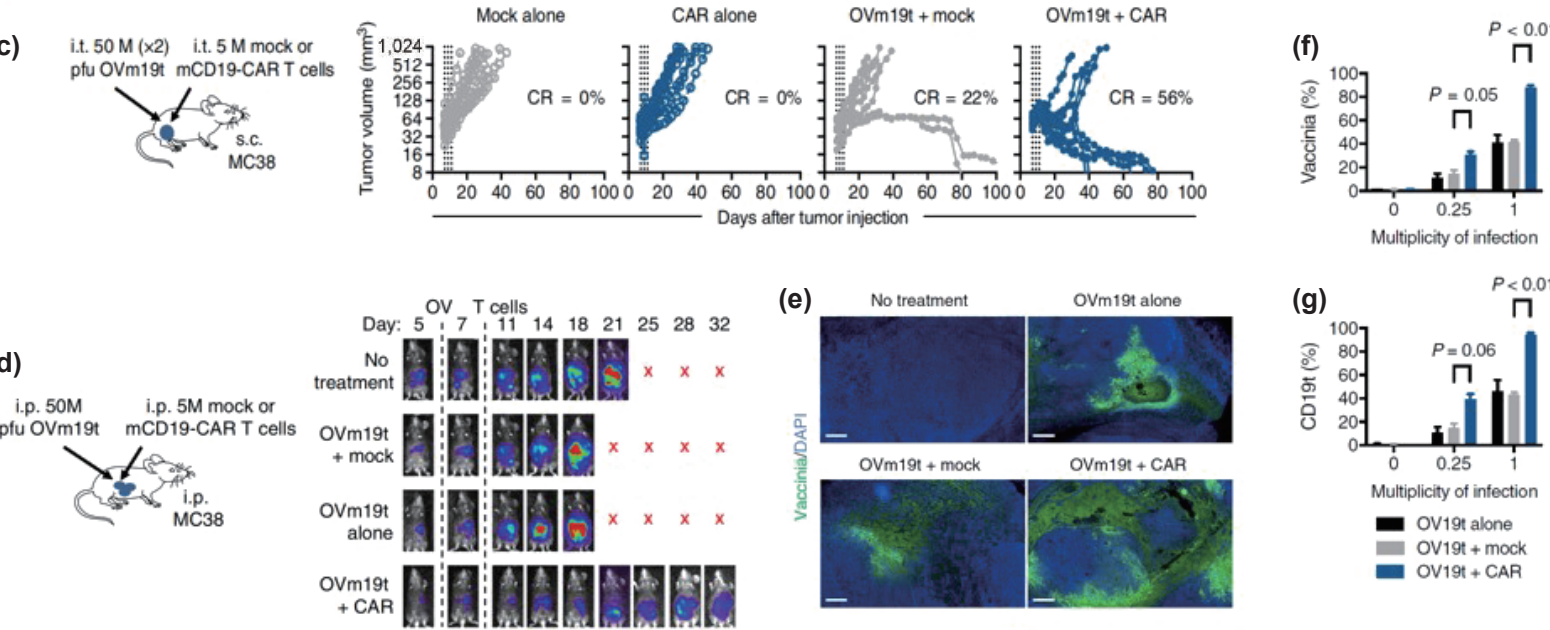

(e)

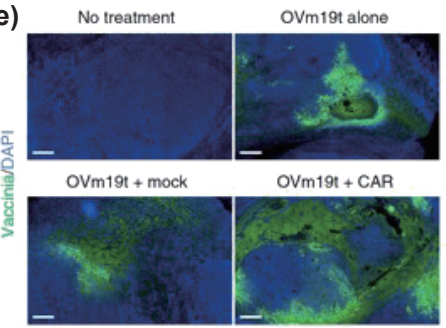

(g)

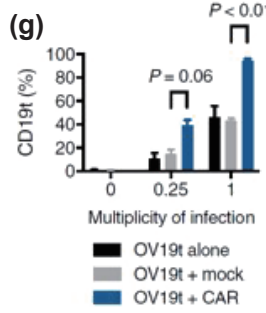

(i)
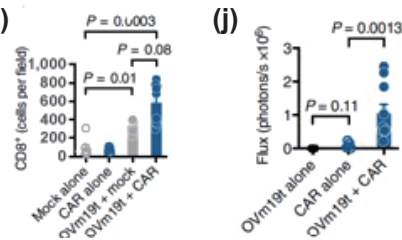

(k)

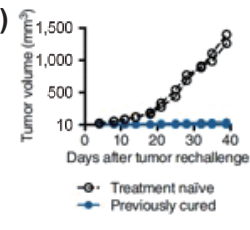

Figure 5 Effective combination immunotherapy using oncolytic viruses to deliver CAR targets to solid tumors. (a) OV effectively delivers CD19t to solid tumors in vitro. Schematic of vaccinia OV [CF33-(SE)hCD19t] showing incorporation of human truncated CD19 (CD19t) under the control of the synthetic early promoter $\left(P_{\mathrm{SE}}\right)$ inserted into the J2R locus and replacing the thymidine kinase (tk) gene (scale bar: $10 \mu \mathrm{m}$ ). (b) Antitumor efficacy of combination therapy of OV19t and CD19-CAR-T cells in human xenograft tumor models. (c) and (d) Antitumor efficacy of combination therapy of OVm19t and mCD19-CAR-T cells in an immunocompetent murine syngeneic tumor model. (e)-(g) CD19-CAR-T cell-mediated tumor killing promotes the viral particle release and infection of tumor cells (scale bar in (e): 500 $\mu \mathrm{m})$. (h)-(k) OVm19t promotes tumor infiltration of both endogenous T cells and adoptively transferred mCD19-CAR-T cells. Reproduced with permission from Ref. [106], (c) Park, A. K. et al., some rights reserved; exclusive licensee American Association for the Advancement of Science. No claim to original U.S. Government Works 2020.

melanoma. However, more clinical data are needed in this area to broaden the indications for patients with tumors. Furthermore, the relative timing of administering anti-CTLA 4, OVs, anti-PD (L)1, and emerging ICI antibodies is another important area that requires careful observation. Systematic exploration of the effects of ICIs before, simultaneously, or after OVT will improve the design of future clinical trials to improve efficacy and improve patient response rates.

\subsection{Synergistic OVs-mediated radiation therapy}

Ionizing radiation causes cell death by inducing DNA damage, especially double-stranded DNA breakage [123]. Recent preclinical experiments on the effect of OV-mediated radiation therapy have illustrated its dual importance in cancer therapy. On the one hand, radiotherapy promotes a specific form of immunogenic cell death, synergizes with the anti-tumor immunity of OVs, and ultimately augments the therapeutic potential of antitumor immune responses [124]. On the other hand, OVs can sensitize tumor cells to RT [125]. Researchers have combined interferon $\beta$ (IFN $\beta$ )-expressing blister stomatitis virus (types) with $\mathrm{RT}$ in an attempt to treat prostate cancer. Research on subcutaneous PC3 and the heterogeneous transplantation model of the prostate, as well as with the synergy of the RM9 prostate tumor model, demonstrated that administering VSV-IFN $\beta$ combined with RT amplified cancer cell killing. The antitumor mechanism is that RT enhances the oncolytic activity of VSV, resulting in increased VSV replication and an increased CD8 lymphocyte count, thereby enhancing the antitumor activity by weakening the innate antiviral response [126]. In a paper published in the Lancet journal EBioMedicine, Goff et al. evaluated the clinical effect of the Newcastle disease virus (NDV) combined with RT on prolonged survival [127]. According to the results of the survey, the efficacy of NDV combined with RT was dose-dependent, the curative effect was proportional to the radiation dose, and the antitumor therapeutic effect was enhanced by increasing the amount of RT. RT combined with bladder specific soluble tumor adenovirus in bladder cancer cells also has a significant antitumor effect in a dose- and time-dependent manner through the inhibition of cancer cell growth. These results confirmed the synergistic antitumor effect of adenovirus-RT and provided a promising strategy for the treatment of bladder cancer [128]. In summary, the combination of RT with OVs provided a new alternative strategy for cancer treatment with promising prospects. It should be noted that in this combination, selecting increased radiation exposure time and the types of OVs, as well as the duration of treatment, will be important influencing factors.

\section{Challenges and future prospective}

It is clear that various tough problems exist in the field of OVT against cancer hitherto based on the proportion of listed OV drugs, 
even though countless efforts have been made to solve these problems. First, safety is paramount for novel agents, but it cannot be guaranteed in the process of OVT due to the particularities of the virus. OVT is a conscious attempt to selectively convert the integrated tumor into a localized broken and infected TME. Severe adverse events would occur if the virus is distributed throughout the body. Therefore, a precise method should be formulated to enhance the control of viral infection. Moreover, owing to the complicated preparation process of viral agents and the nanoscale size of OVs, the extraction of a pure nontoxic single virus cannot be obtained. Also, there are more obstacles in the OV administration process compared with small-molecule drug delivery systems, such as immune cells and immune factors, as well as in vivo nAbs. Although vast efforts have been made to seek out an OV systemic delivery model against multiple malignant tumors as reviewed in this article, none of them are so ideal that they could become a mainstream clinical cancer treatment method. In addition, the production of live OV agents is more complicated than that of a common vaccine and small molecular drugs. A more stringent quality standard is demanded during the manufacturing, storage, and use of $\mathrm{OV}$ agents, which further increases the difficulty of making OVT pervasive.

Despite the aforementioned obstacles, we are confident in the efficacy of OVT. Owing to its centurial development history, therapy using viruses at its forefront continues to improve gradually. On one hand, various systemic delivery models improve the druggability of OVs, including their stability, safety, and efficiency, all of which cause OVs to overcome biological delivery obstacles and obtain an acquired tumor tropism. However, synergistic therapy has undoubtedly become a trend in preclinical and clinical studies. All types of traditional monotherapy (i.e., chemotherapy, RT, thermotherapy, photodynamic therapy, or immunotherapy), still have various drawbacks, even though OVT remains an imperfect pathway against cancers, as mentioned above. Synergistic OV-mediated therapies not only offset each other's defects, but may also achieve a "one plus one is greater than two" effect in cancer treatment. In addition, recent reports have demonstrated that some patients with cancer went into remission being infected with COVID-19. Although little evidence proving the correlation between the recovery of cancer and COVID-19 infection has been provided to date, these occurrences have undoubtedly attracted more attention to OVs and OVT. In summary, with the aid of more rational design and further exploration, OVT may develop into a highly effective treatment against cancer in the near future.

\section{Conclusions}

Currently, OVT is a novel immunotherapy often used in cancer treatment, and has again caused a stir in the scientific community owing to the COVID-19 pandemic and the publication of a paper that demonstrated the efficacy of combining OVT and different traditional anticancer therapeutic modalities. After collecting a considerable amount of preclinical and clinical data and novel scientific reports, we have analyzed and outlined the practical development conditions of OVT from several aspects. The mature mechanisms of OVs against tumors have been explored by many researchers in the past few years and outlined in our paper. Next, we illustrated the practical druggability of OVs and their ability to treat cancer in patients with advanced cancer based on several types of clinical data, and subsequently clarified the superiority of the systemic delivery system by analyzing and comparing the properties of existing administration methods. We also described the OVs systemic administration model in detail. Furthermore, we shed light on the combination of OVT and traditional therapies. A creative concept has undoubtedly been put forward, and we believe that an improved system for OVT should be established soon, given the iteration of science and technology.

\section{Acknowledgments}

This work was supported by the National Key R\&D Program of China (No. 2019YFC1316104), the National Natural Science Foundation of China (Nos. 81871960, 82073368, and 82073777), Liaoning Revitalization Talents Program (Nos. XLYC2007071 and XLYC1808017), China Postdoctoral Science Foundation (No. 2020M680986), and General Project of Liaoning Provincial Department of Education (No. JKZ0927).

\section{References}

[1] Advani, S. J.; Chung, S. M.; Yan, S. Y.; Gillespie, G. Y.; Markert, J. M.; Whitley, R. J.; Roizman, B.; Weichselbaum, R. R. Replication-competent, nonneuroinvasive genetically engineered herpes virus is highly effective in the treatment of therapy-resistant experimental human tumors. Cancer Res. 1999, 59, 2055-2058.

[2] Skoetz, N.; Will, A.; Monsef, I.; Brillant, C.; Engert, A.; von Tresckow, B. Comparison of first-line chemotherapy including escalated BEACOPP versus chemotherapy including ABVD for people with early unfavourable or advanced stage Hodgkin lymphoma. Cochrane Database Syst. Rev. 2017, 5, Cd007941.

[3] Watanabe, D.; Goshima, F. Oncolytic virotherapy by HSV. Adv. Exp. Med. Biol. 2018, 1045, 63-84.

[4] Masoud, S. J.; Hu, J. B.; Beasley, G. M.; Stewart IV, J. H.; Mosca, P. J. Efficacy of talimogene laherparepvec (T-VEC) therapy in patients with in-transit melanoma metastasis decreases with increasing lesion size. Ann. Surg. Oncol. 2019, 26, 4633-4641.

[5] Bai, Y.; Hui, P.; Du, X. Y.; Su, X. Updates to the antitumor mechanism of oncolytic virus. Thorac. Cancer 2019, 10, 1031-1035.

[6] Lee, P.; Gujar, S. Potentiating prostate cancer immunotherapy with oncolytic viruses. Nat. Rev. Urol. 2018, 15, 235-250.

[7] Lv, P.; Liu, X.; Chen, X. M.; Liu, C.; Zhang, Y.; Chu, C. C.; Wang, J. Q.; Wang, X. Y.; Chen, X. Y.; Liu, G. Genetically engineered cell membrane nanovesicles for oncolytic adenovirus delivery: A versatile platform for cancer virotherapy. Nano Lett. 2019, 19, 2993-3001.

[8] Bommareddy, P. K.; Shettigar, M.; Kaufman, H. L. Integrating oncolytic viruses in combination cancer immunotherapy. Nat. Rev. Immunol. 2018, 18, 498-513.

[9] Smith, J. S.; Xu, Z. L.; Tian, J.; Palmer, D. J.; Ng, P.; Byrnes, A. P. The role of endosomal escape and mitogen-activated protein kinases in adenoviral activation of the innate immune response. PLoS One 2011, 6, e26755.

[10] Waddington, S. N.; McVey, J. H.; Bhella, D.; Parker, A. L.; Barker, K.; Atoda, H.; Pink, R.; Buckley, S. M. K.; Greig, J. A.; Denby, L. et al. Adenovirus serotype 5 hexon mediates liver gene transfer. Cell 2008, 132, 397-409.

[11] Atasheva, S.; Yao, J.; Shayakhmetov, D. M. Innate immunity to adenovirus: Lessons from mice. FEBS Lett. 2019, 593, 3461-3483.

[12] Taipale, K.; Liikanen, I.; Juhila, J.; Turkki, R.; Tähtinen, S.; Kankainen, M.; Vassilev, L.; Ristimäki, A.; Koski, A.; Kanerva, A. et al. Chronic activation of innate immunity correlates with poor prognosis in cancer patients treated with oncolytic adenovirus. Mol. Ther. 2016, 24, 175-183.

[13] Brown, M. C.; Holl, E. K.; Boczkowski, D.; Dobrikova, E.; Mosaheb, M.; Chandramohan, V.; Bigner, D. D.; Gromeier, M.; Nair, S. K. Cancer immunotherapy with recombinant poliovirus induces IFN-dominant activation of dendritic cells and tumor antigen-specific CTLs. Sci. Transl. Med. 2017, 9, eaan4220.

[14] Guo, G.; Gong, K.; Wohlfeld, B.; Hatanpaa, K. J.; Zhao, D. W.; Habib, A. A. Ligand-independent EGFR signaling. Cancer Res. 2015, 75, 3436-3441.

[15] Ilkow, C. S.; Marguerie, M.; Batenchuk, C.; Mayer, J.; Ben Neriah, D.; Cousineau, S.; Falls, T.; Jennings, V. A.; Boileau, M.; Bellamy, 
D. et al. Reciprocal cellular cross-talk within the tumor microenvironment promotes oncolytic virus activity. Nat. Med. 2015, 21, 530-536.

[16] Arulanandam, R.; Batenchuk, C.; Angarita, F. A.; Ottolino-Perry, K.; Cousineau, S.; Mottashed, A.; Burgess, E.; Falls, T. J.; De Silva, N.; Tsang, J. et al. VEGF-mediated induction of PRD1-BF1/blimp1 expression sensitizes tumor vasculature to oncolytic virus infection. Cancer Cell 2015, 28, 210-224.

[17] Shmulevitz, M.; Marcato, P.; Lee, P. W. K. Unshackling the links between reovirus oncolysis, Ras signaling, translational control, and cancer. Oncogene 2005, 24, 7720-7728.

[18] Martin, N. T.; Bell, J. C. Oncolytic virus combination therapy: Killing one bird with two stones. Mol. Ther. 2018, 26, 1414-1422.

[19] Chiappinelli, K. B.; Strissel, P. L.; Desrichard, A.; Li, H. L.; Henke, C.; Akman, B.; Hein, A.; Rote, N. S.; Cope, L. M.; Snyder, A. et al. Inhibiting DNA methylation causes an interferon response in cancer via dsRNA including endogenous retroviruses. Cell 2015, 162, 974-986.

[20] Kaufman, H. L.; Kohlhapp, F. J.; Zloza, A. Oncolytic viruses: A new class of immunotherapy drugs. Nat. Rev. Drug Discov. 2015, 14, 642-662.

[21] Gujar, S.; Pol, J. G.; Kim, Y.; Lee, P. W.; Kroemer, G. Antitumor benefits of antiviral immunity: An underappreciated aspect of oncolytic virotherapies. Trends Immunol. 2018, 39, 209-221.

[22] Gujar, S. A.; Pan, D.; Marcato, P.; Garant, K. A.; Lee, P. W. K. Oncolytic virus-initiated protective immunity against prostate cancer. Mol. Ther. 2011, 19, 797-804.

[23] Lucas, T.; Abraham, D.; Untergasser, G.; Zins, K.; Hofer, E.; Gunsilius, E.; Aharinejad, S. Adenoviral-mediated endothelial precursor cell delivery of soluble cd115 suppresses human prostate cancer xenograft growth in mice. Stem Cells 2009, 27, 2342-2352.

[24] Mogensen, T. H. Pathogen recognition and inflammatory signaling in innate immune defenses. Clin. Microbiol. Rev. 2009, 22, 240-273.

[25] Kepp, O.; Senovilla, L.; Vitale, I.; Vacchelli, E.; Adjemian, S.; Agostinis, P.; Apetoh, L.; Aranda, F.; Barnaba, V.; Bloy, N. et al. Consensus guidelines for the detection of immunogenic cell death. Oncoimmunology 2014, 3, e955691.

[26] Getts, D. R.; Chastain, E. M. L.; Terry, R. L.; Miller, S. D. Virus infection, antiviral immunity, and autoimmunity. Immunol. Rev. 2013, 255, 197-209.

[27] Dörner, T.; Radbruch, A. Antibodies and B cell memory in viral immunity. Immunity 2007, 27, 384-392.

[28] Bhattacharya, P.; Budnick, I.; Singh, M.; Thiruppathi, M.; Alharshawi, K.; Elshabrawy, H.; Holterman, M. J.; Prabhakar, B. S. Dual role of GM-CSF as a pro-inflammatory and a regulatory cytokine: Implications for immune therapy. J. Interferon Cytokine Res. 2015, 35, 585-599.

[29] Tähtinen, S.; Blattner, C.; Vähä-Koskela, M.; Saha, D.; Siurala, M.; Parviainen, S.; Utikal, J.; Kanerva, A.; Umansky, V.; Hemminki, A. T-cell therapy enabling adenoviruses coding for IL2 and TNFo induce systemic immunomodulation in mice with spontaneous melanoma. J. Immunother. 2016, 39, 343-354.

[30] Passer, B. J.; Cheema, T.; Wu, S.; Wu, C. L.; Rabkin, S. D.; Martuza, R. L. Combination of vinblastine and oncolytic herpes simplex virus vector expressing IL-12 therapy increases antitumor and antiangiogenic effects in prostate cancer models. Cancer Gene Ther. 2013, 20, 17-24.

[31] Zamarin, D.; Wolchok, J. D. Potentiation of immunomodulatory antibody therapy with oncolytic viruses for treatment of cancer. Mol. Ther. Oncolytics 2014, 1, 14004.

[32] Choi, J. W.; Lee, Y. S.; Yun, C. O.; Kim, S. W. Polymeric oncolytic adenovirus for cancer gene therapy. J. Control. Release 2015, 219, 181-191.

[33] Andtbacka, R. H. I.; Amatruda, T.; Nemunaitis, J.; Zager, J. S.; Walker, J.; Chesney, J. A.; Liu, K. T.; Hsu, C. P.; Pickett, C. A.; Mehnert, J. M. Biodistribution, shedding, and transmissibility of the oncolytic virus talimogene laherparepvec in patients with melanoma. EBioMedicine 2019, 47, 89-97.

[34] Hirooka, Y.; Kasuya, H.; Ishikawa, T.; Kawashima, H.; Ohno, E.;
Villalobos, I. B.; Naoe, Y.; Ichinose, T.; Koyama, N.; Tanaka, M et al. A phase I clinical trial of EUS-guided intratumoral injection of the oncolytic virus, HF10 for unresectable locally advanced pancreatic cancer. BMC Cancer 2018, 18, 596.

[35] Mahalingam, D.; Wilkinson, G. A.; Eng, K. H.; Fields, P.; Raber, P.; Moseley, J. L.; Cheetham, K.; Coffey, M.; Nuovo, G.; Kalinski, P. et al. Pembrolizumab in combination with the oncolytic virus pelareorep and chemotherapy in patients with advanced pancreatic adenocarcinoma: A phase Ib study. Clin. Cancer Res. 2020, 26, 71-81.

[36] Packiriswamy, N.; Upreti, D.; Zhou, Y. M.; Khan, R.; Miller, A. Diaz, R. M.; Rooney, C. M.; Dispenzieri, A.; Peng, K. W.; Russell, S. J. Oncolytic measles virus therapy enhances tumor antigenspecific T-cell responses in patients with multiple myeloma Leukemia 2020, 34, 3310-3322.

[37] García, M.; Moreno, R.; Gil-Martin, M.; Cascallò, M.; de Olza, M. O.; Cuadra, C.; Piulats, J. M.; Navarro, V.; Domenech, M.; Alemany, R. et al. A phase 1 trial of oncolytic adenovirus ICOVIR5 administered intravenously to cutaneous and uveal melanoma patients. Hum. Gene Ther. 2019, 30, 352-364.

[38] Müller, L. M. E.; Holmes, M.; Michael, J. L.; Scott, G. B.; West, E. J.; Scott, K. J.; Parrish, C.; Hall, K.; Stäble, S.; Jennings, V. A. et al. Plasmacytoid dendritic cells orchestrate innate and adaptive antitumor immunity induced by oncolytic coxsackievirus A21. J. Immunother. Cancer 2019, 7, 164.

[39] Annels, N. E.; Mansfield, D.; Arif, M.; Ballesteros-Merino, C.; Simpson, G. R.; Denyer, M.; Sandhu, S. S.; Melcher, A. A.; Harrington, K. J.; Davies, B. et al. Phase I trial of an ICAM-1targeted immunotherapeutic-coxsackievirus A21 (CVA21) as an oncolytic agent against non muscle-invasive bladder cancer. Clin Cancer Res. 2019, 25, 5818-5831.

[40] Kurokawa, C.; Iankov, I. D.; Anderson, S. K.; Aderca, I.; Leontovich, A. A.; Maurer, M. J.; Oberg, A. L.; Schroeder, M. A. Giannini, C.; Greiner, S. M. et al. Constitutive interferon pathway activation in tumors as an efficacy determinant following oncolytic virotherapy. J. Natl. Cancer Inst. 2018, 110, 1123-1132.

[41] Lauer, U. M.; Schell, M.; Beil, J.; Berchtold, S.; Koppenhöfer, U.; Glatzle, J.; Königsrainer, A.; Möhle, R.; Nann, D.; Fend, F. et al Phase I study of oncolytic vaccinia virus GL-ONC1 in patients with peritoneal carcinomatosis. Clin. Cancer Res. 2018, 24, 4388-4398.

[42] Pascual-Pasto, G.; Bazan-Peregrino, M.; Olaciregui, N. G.; Restrepo-Perdomo, C. A.; Mato-Berciano, A.; Ottaviani, D.; Weber, K.; Correa, G.; Paco, S.; Vila-Ubach, M. et al. Therapeutic targeting of the RB1 pathway in retinoblastoma with the oncolytic adenovirus VCN-01. Sci. Transl. Med. 2019, 11, eaat9321.

[43] Lang, F. F.; Conrad, C.; Gomez-Manzano, C.; Yung, W. K. A.; Sawaya, R.; Weinberg, J. S.; Prabhu, S. S.; Rao, G.; Fuller, G. N.; Aldape, K. D. et al. Phase I study of DNX-2401 (Delta-24-RGD) oncolytic adenovirus: Replication and immunotherapeutic effects in recurrent malignant glioma. J. Clin. Oncol. 2018, 36, 1419-1427.

[44] Streby, K. A.; Currier, M. A.; Triplet, M.; Ott, K.; Dishman, D. J.; Vaughan, M. R.; Ranalli, M. A.; Setty, B.; Skeens, M. A.; Whiteside, S. et al. First-in-human intravenous seprehvir in young cancer patients: A phase 1 clinical trial. Mol. Ther. 2019, 27, 1930-1938.

[45] Jonker, D. J.; Tang, P. A.; Kennecke, H.; Welch, S. A.; Cripps, M. C.; Asmis, T.; Chalchal, H.; Tomiak, A.; Lim, H.; Ko, Y. J. et al. A randomized phase II study of FOLFOX6/bevacizumab with or without pelareorep in patients with metastatic colorectal cancer: IND. 210, a Canadian cancer trials group trial. Clin. Colorectal Cancer 2018, 17, 231-239.e7.

[46] Machiels, J. P.; Salazar, R.; Rottey, S.; Duran, I.; Dirix, L.; Geboes, K.; Wilkinson-Blanc, C.; Pover, G.; Alvis, S.; Champion, B. et al. A phase 1 dose escalation study of the oncolytic adenovirus enadenotucirev, administered intravenously to patients with epithelial solid tumors (EVOLVE). J. Immunother. Cancer 2019, 7, 20.

[47] Packiam, V. T.; Lamm, D. L.; Barocas, D. A.; Trainer, A.; Fand, B.; Davis III, R. L.; Clark, W.; Kroeger, M.; Dumbadze, I.; Chamie, $\mathrm{K}$. et al. An open label, single-arm, phase II multicenter study of the safety and efficacy of CG0070 oncolytic vector regimen in 
patients with BCG-unresponsive non-muscle-invasive bladder cancer: Interim results. Urol. Oncol.:Semin. Orig. Investig. 2018, 36, 440-447.

[48] Parakrama, R.; Fogel, E.; Chandy, C.; Augustine, T.; Coffey, M.; Tesfa, L.; Goel, S.; Maitra, R. Immune characterization of metastatic colorectal cancer patients post reovirus administration. BMC Cancer 2020, 20, 569.

[49] Bradbury, P. A.; Morris, D. G.; Nicholas, G.; Tu, D. S.; Tehfe, M.; Goffin, J. R.; Shepherd, F. A.; Gregg, R. W.; Rothenstein, J.; Lee, C. et al. Canadian cancer trials group (CCTG) IND211: A randomized trial of pelareorep (Reolysin) in patients with previously treated advanced or metastatic non-small cell lung cancer receiving standard salvage therapy. Lung Cancer 2018, 120, 142-148.

[50] Reid, E. G.; Looney, D.; Maldarelli, F.; Noy, A.; Henry, D.; Aboulafia, D.; Ramos, J. C.; Sparano, J.; Ambinder, R. F.; Lee, J. et al. Safety and efficacy of an oncolytic viral strategy using bortezomib with ICE/R in relapsed/refractory HIV-positive lymphomas. Blood Adv. 2018, 2, 3618-3626.

[51] Schenk, E. L.; Mandrekar, S. J.; Dy, G. K.; Aubry, M. C.; Tan, A. D.; Dakhil, S. R.; Sachs, B. A.; Nieva, J. J.; Bertino, E.; Hann, C. L. et al. A randomized double-blind phase II study of the Seneca valley virus (NTX-010) versus placebo for patients with extensivestage SCLC (ES SCLC) who were stable or responding after at least four cycles of platinum-based chemotherapy: North central cancer treatment group (Alliance) N0923 study. J. Thorac. Oncol. 2020, 15, 110-119.

[52] Kiyohara, E.; Tanemura, A.; Nishioka, M.; Yamada, M.; Tanaka, A.; Yokomi, A.; Saito, A.; Sakura, K.; Nakajima, T.; Myoui, A. et al. Intratumoral injection of hemagglutinating virus of Japanenvelope vector yielded an antitumor effect for advanced melanoma: A phase I/IIa clinical study. Cancer Immunol., Immunother. 2020, 69, 1131-1140.

[53] Fujita, K.; Kato, T.; Hatano, K.; Kawashima, A.; Ujike, T.; Uemura, M.; Imamura, R.; Okihara, K.; Ukimura, O.; Miki, T. et al. Intratumoral and s. c. injection of inactivated hemagglutinating virus of Japan envelope (GEN0101) in metastatic castrationresistant prostate cancer. Cancer Sci. 2020, 111, 1692-1698.

[54] Bernstein, V.; Ellard, S. L.; Dent, S. F.; Tu, D.; Mates, M.; DhesyThind, S. K.; Panasci, L.; Gelmon, K. A.; Salim, M.; Song, X. et al. A randomized phase II study of weekly paclitaxel with or without pelareorep in patients with metastatic breast cancer: Final analysis of Canadian cancer trials group IND. 213. Breast Cancer Res. Treat. 2018, 167, 485-493.

[55] Chesney, J.; Puzanov, I.; Collichio, F.; Singh, P.; Milhem, M. M.; Glaspy, J.; Hamid, O.; Ross, M.; Friedlander, P.; Garbe, C. et al. Randomized, open-label phase II study evaluating the efficacy and safety of talimogene laherparepvec in combination with ipilimumab versus ipilimumab alone in patients with advanced, unresectable melanoma. J. Clin. Oncol. 2018, 36, 1658-1667.

[56] Tejada, S.; Alonso, M.; Patiño, A.; Fueyo, J.; Gomez-Manzano, C.; Diez-Valle, R. Phase I trial of DNX-2401 for diffuse intrinsic pontine glioma newly diagnosed in pediatric patients. Neurosurgery 2018, 83, 1050-1056.

[57] Chesney, J.; Awasthi, S.; Curti, B.; Hutchins, L.; Linette, G.; Triozzi, P.; Tan, M. C. B.; Brown, R. E.; Nemunaitis, J.; Whitman, E. et al. Phase IIIb safety results from an expanded-access protocol of talimogene laherparepvec for patients with unresected, stage IIIBIVM1c melanoma. Melanoma Res. 2018, 28, 44-51.

[58] Macedo, N.; Miller, D. M.; Haq, R.; Kaufman, H. L. Clinical landscape of oncolytic virus research in 2020. J. Immunother. Cancer 2020, 8, e001486.

[59] Zheng, M. J.; Huang, J. H.; Tong, A. P.; Yang, H. Oncolytic viruses for cancer therapy: Barriers and recent advances. Mol. Ther. Oncolytics 2019, 15, 234-247.

[60] Smith, E.; Breznik, J.; Lichty, B. D. Strategies to enhance viral penetration of solid tumors. Hum. Gene Ther. 2011, 22, $1053-1060$

[61] Roy, D. G.; Bell, J. C. Cell carriers for oncolytic viruses: Current challenges and future directions. Oncolytic Virother 2013,2, $47-56$.
[62] Bridle, B. W.; Stephenson, K. B.; Boudreau, J. E.; Koshy, S.; Kazdhan, N.; Pullenayegum, E.; Brunellière, J.; Bramson, J. L.; Lichty, B. D.; Wan, Y. H. Potentiating cancer immunotherapy using an oncolytic virus. Mol. Ther. 2010, 18, 1430-1439.

[63] Hill, C.; Carlisle, R. Achieving systemic delivery of oncolytic viruses. Expert Opin. Drug Deliv. 2019, 16, 607-620.

[64] Guimarães-Camboa, N.; Cattaneo, P.; Sun, Y. F.; Moore-Morris, T.; Gu, Y. S.; Dalton, N. D.; Rockenstein, E.; Masliah, E.; Peterson, K. L.; Stallcup, W. B. et al. Pericytes of multiple organs do not behave as mesenchymal stem cells in vivo. Cell Stem Cell 2017, 20, 345-359.e5.

[65] Hadryś, A.; Sochanik, A.; McFadden, G.; Jazowiecka-Rakus, J. Mesenchymal stem cells as carriers for systemic delivery of oncolytic viruses. Eur. J. Pharmacol. 2020, 874, 172991

[66] Abdallah, B. M.; Kassem, M. The use of mesenchymal (skeletal) stem cells for treatment of degenerative diseases: Current status and future perspectives. J. Cell. Physiol. 2009, 218, 9-12.

[67] Cheng, X. F.; Zhang, G. Y.; Zhang, L.; Hu, Y.; Zhang, K.; Sun, X J.; Zhao, C. Q.; Li, H.; Li, Y. M.; Zhao, J. Mesenchymal stem cells deliver exogenous mir-21 via exosomes to inhibit nucleus pulposus cell apoptosis and reduce intervertebral disc degeneration. J. Cell. Mol. Med. 2018, 22, 261-276.

[68] Wang, B.; Yao, K.; Huuskes, B. M.; Shen, H. H.; Zhuang, J. L.; Godson, C.; Brennan, E. P.; Wilkinson-Berka, J. L.; Wise, A. F.; Ricardo, S. D. Mesenchymal stem cells deliver exogenous MicroRNA-let7c via exosomes to attenuate renal fibrosis. Mol. Ther. 2016, 24, 1290-1301.

[69] Na, Y. J.; Nam, J. P.; Hong, J.; Oh, E.; Shin, H. C.; Kim, H. S.; Kim, S. W.; Yun, C. O. Systemic administration of human mesenchymal stromal cells infected with polymer-coated oncolytic adenovirus induces efficient pancreatic tumor homing and infiltration. J. Control. Release 2019, 305, 75-88.

[70] Dapkute, D.; Steponkiene, S.; Bulotiene, D.; Saulite, L.; Riekstina, U.; Rotomskis, R. Skin-derived mesenchymal stem cells as quantum dot vehicles to tumors. Int. J. Nanomedicine 2017, 12, 8129-8142.

[71] Liu, Y.; Ye, T.; Maynard, J.; Akbulut, H.; Deisseroth, A. Engineering conditionally replication-competent adenoviral vectors carrying the cytosine deaminase gene increases the infectivity and therapeutic effect for breast cancer gene therapy. Cancer Gene Ther. 2006, 13, 346-356.

[72] Long, G. V.; Trefzer, U.; Davies, M. A.; Kefford, R. F.; Ascierto, P. A.; Chapman, P. B.; Puzanov, I.; Hauschild, A.; Robert, C.; Algazi, A. et al. Dabrafenib in patients with Val600Glu or Val600Lys BRAF-mutant melanoma metastatic to the brain (BREAK-MB): A multicentre, open-label, phase 2 trial. Lancet Oncol. 2012, 13, 1087-1095.

[73] Harrington, K. J.; Puzanov, I.; Hecht, J. R.; Hodi, F. S.; Szabo, Z.; Murugappan, S.; Kaufman, H. L. Clinical development of talimogene laherparepvec (T-VEC): A modified herpes simplex virus type-1-derived oncolytic immunotherapy. Expert Rev. Anticancer Ther. 2015, 15, 1389-1403.

[74] Du, W.; Seah, I.; Bougazzoul, O.; Choi, G.; Meeth, K.; Bosenberg, M. W.; Wakimoto, H.; Fisher, D.; Shah, K. Stem cell-released oncolytic herpes simplex virus has therapeutic efficacy in brain metastatic melanomas. P. Natl. Acad. Sci. USA 2017, 114, E6157-E6165.

[75] Yang, F. J.; Wu, L. N.; Xu, W. X.; Liu, Y.; Zhen, L. M.; Ning, G.; Song, J.; Jiao, Q.; Zheng, Y. Y.; Chen, T. T. et al. Diverse effects of the NTCP p. Ser267Phe variant on disease progression during chronic HBV infection and on HBV pres1 variability. Front. Cell. Infect. Microbiol. 2019, 9, 18.

[76] Cui, S. X.; Zhang, H. L.; Xu, W. F.; Qu, X. J. 13F-1, a novel 5fluorouracil prodrug containing an Asn-Gly-Arg $\left(\mathrm{NO}_{2}\right) \mathrm{COOCH}_{3}$ tripeptide, inhibits human colonic carcinoma growth by targeting Aminopeptidase N (APN/CD13). Eur. J. Pharmacol. 2014, 734, $50-59$.

[77] Fusciello, M.; Fontana, F.; Tähtinen, S.; Capasso, C.; Feola, S.; Martins, B.; Chiaro, J.; Peltonen, K.; Ylösmäki, L.; Ylösmäki, E. et al. Artificially cloaked viral nanovaccine for cancer immunotherapy. Nat. Commun. 2019, 10, 5747. 
[78] Rojas, L. A.; Condezo, G. N.; Moreno, R.; Fajardo, C. A.; AriasBadia, M.; San Martín, C.; Alemany, R. Albumin-binding adenoviruses circumvent pre-existing neutralizing antibodies upon systemic delivery. J. Control. Release 2016, 237, 78-88.

[79] Mato-Berciano, A.; Morgado, S.; Maliandi, M. V.; Farrera-Sal, M.; Gimenez-Alejandre, M.; Ginestà, M. M.; Moreno, R.; TorresManjon, S.; Moreno, P.; Arias-Badia, M. et al. Oncolytic adenovirus with hyaluronidase activity that evades neutralizing antibodies: VCN-11. J. Control. Release 2021, 332, 517-528.

[80] Guedan, S.; Rojas, J. J.; Gros, A.; Mercade, E.; Cascallo, M.; Alemany, R. Hyaluronidase expression by an oncolytic adenovirus enhances its intratumoral spread and suppresses tumor growth. Mol. Ther. 2010, 18, 1275-1283.

[81] Schmid, M.; Ernst, P.; Honegger, A.; Suomalainen, M.; Zimmermann, M.; Braun, L.; Stauffer, S.; Thom, C.; Dreier, B.; Eibauer, M. et al. Adenoviral vector with shield and adapter increases tumor specificity and escapes liver and immune control. Nat. Commun. 2018, 9, 450.

[82] Rangaswamy, U. S.; Cotter, C. R.; Cheng, X.; Jin, H.; Chen, Z. $\mathrm{CD} 55$ is a key complement regulatory protein that counteracts complement-mediated inactivation of Newcastle Disease Virus. $J$. Gen. Virol. 2016, 97, 1765-1770.-.

[83] Brunetti-Pierri, N.; Palmer, D. J.; Beaudet, A. L.; Carey, K. D.; Finegold, M.; Ng, P. Acute toxicity after high-dose systemic injection of helper-dependent adenoviral vectors into nonhuman primates. Hum. Gene Ther. 2004, 15, 35-46.

[84] Beebe, D. P.; Cooper, N. R. Neutralization of vesicular stomatitis virus (VSV) by human complement requires a natural IgM antibody present in human serum. J. Immunol. 1981, 126, 1562-1568.

[85] Tesfay, M. Z.; Ammayappan, A.; Federspiel, M. J.; Barber, G. N.; Stojdl, D.; Peng, K. W.; Russell, S. J. Vesiculovirus neutralization by natural IgM and complement. J. Virol. 2014, 88, 6148-6157.

[86] Atasheva, S.; Emerson, C. C.; Yao, J.; Young, C.; Stewart, P. L.; Shayakhmetov, D. M. Systemic cancer therapy with engineered adenovirus that evades innate immunity. Sci. Transl. Med. 2020, 12, eabc6659.

[87] Bradley, R. R.; Lynch, D. M.; Iampietro, M. J.; Borducchi, E. N.; Barouch, D. H. Adenovirus serotype 5 neutralizing antibodies target both hexon and fiber following vaccination and natural infection. J. Virol. 2012, 86, 625-629.

[88] Bradley, R. R.; Maxfield, L. F.; Lynch, D. M.; Iampietro, M. J.; Borducchi, E. N.; Barouch, D. H. Adenovirus serotype 5-specific neutralizing antibodies target multiple hexon hypervariable regions. J. Virol. 2012, 86, 1267-1272.

[89] Pipperger, L.; Koske, I.; Wild, N.; Müllauer, B.; Krenn, D.; Stoiber, H.; Wollmann, G.; Kimpel, J.; von Laer, D.; Bánki, Z. Xenoantigendependent complement-mediated neutralization of lymphocytic choriomeningitis virus glycoprotein-pseudotyped vesicular stomatitis virus in human serum. J. Virol. 2019, 93, e00567-19.

[90] Howard, F.; Muthana, M. Designer nanocarriers for navigating the systemic delivery of oncolytic viruses. Nanomedicine 2020, 15, 93-110.

[91] Huang, L. L.; Li, X.; Zhang, J. F.; Zhao, Q. R.; Zhang, M. J.; Liu, A. A.; Pang, D. W.; Xie, H. Y. MnCaCs-biomineralized oncolytic virus for bimodal imaging-guided and synergistically enhanced anticancer therapy. Nano Lett. 2019, 19, 8002-8009.

[92] Almstätter, I.; Mykhaylyk, O.; Settles, M.; Altomonte, J.; Aichler, M.; Walch, A.; Rummeny, E. J.; Ebert, O.; Plank, C.; Braren, R. Characterization of magnetic viral complexes for targeted delivery in oncology. Theranostics 2015, 5, 667-685.

[93] Garofalo, M.; Bellato, F.; Magliocca, S.; Malfanti, A.; Kuryk, L.; Rinner, B.; Negro, S.; Salmaso, S.; Caliceti, P.; Mastrotto, F. Polymer coated oncolytic adenovirus to selectively target hepatocellular carcinoma cells. Pharmaceutics 2021, 13, 949.

[94] Hill, C.; Grundy, M.; Bau, L.; Wallington, S.; Balkaran, J.; Ramos, V.; Fisher, K.; Seymour, L.; Coussios, C.; Carlisle, R. Polymer stealthing and mucin-1 retargeting for enhanced pharmacokinetics of an oncolytic vaccinia virus. Mol. Ther. Oncolytics 2021, 21, $47-61$.

[95] Lou, X. Y.; Chen, Z. C.; He, Z. G.; Sun, M. C.; Sun, J. Bacteria- mediated synergistic cancer therapy: Small microbiome has a big hope. Nano-Micro Lett. 2021, 13, 37.

[96] Harrington, K. J.; Kong, A.; Mach, N.; Chesney, J. A.; Fernandez, B. C.; Rischin, D.; Cohen, E. E. W.; Radcliffe, H. S.; Gumuscu, B.; Cheng, J. et al. Talimogene laherparepvec and pembrolizumab in recurrent or metastatic squamous cell carcinoma of the head and neck (MASTERKEY-232): A multicenter, phase 1b study. Clin. Cancer Res. 2020, 26, 5153-5161.

[97] Kelly, C. M.; Antonescu, C. R.; Bowler, T.; Munhoz, R.; Chi, P.; Dickson, M. A.; Gounder, M. M.; Keohan, M. L.; Movva, S.; Dholakia, R. et al. Objective response rate among patients with locally advanced or metastatic sarcoma treated with talimogene laherparepvec in combination with pembrolizumab: A phase 2 clinical trial. JAMA Oncol. 2020, 6, 402-408.

[98] Sun, L. L.; Funchain, P.; Song, J. M.; Rayman, P.; Tannenbaum, C.; Ko, J.; Mcnamara, M.; Diaz-Montero, C. M.; Gastman, B. Talimogene laherparepvec combined with anti-PD-1 based immunotherapy for unresectable stage III-IV melanoma: A case series. J. Immunother. Cancer 2018, 6, 36.

[99] Ribas, A.; Dummer, R.; Puzanov, I.; VanderWalde, A.; Andtbacka, R. H. I.; Michielin, O.; Olszanski, A. J.; Malvehy, J.; Cebon, J.; Fernandez, E. et al. Oncolytic virotherapy promotes intratumoral T cell infiltration and improves Anti-PD-1 immunotherapy. Cell 2017, 170, 1109-1119.e10.

[100] Soliman, H.; Hogue, D.; Han, H.; Mooney, B.; Costa, R.; Lee, M. C.; Niell, B.; Williams, A.; Chau, A.; Falcon, S. et al. A phase I trial of talimogene laherparepvec in combination with neoadjuvant chemotherapy for the treatment of nonmetastatic triple-negative breast cancer. Clin. Cancer Res. 2021, 27, 1012.

[101] Wang, L.; Ning, J. F.; Wakimoto, H.; Wu, S. L.; Wu, C. L.; Humphrey, M. R.; Rabkin, S. D.; Martuza, R. L. Oncolytic herpes simplex virus, and PI3K inhibitor BKM120 synergize to promote killing of prostate cancer stem-like cells. Mol. Ther. Oncolytics 2019, 13, 58-66.

[102] Khuri, F. R.; Nemunaitis, J.; Ganly, I.; Arseneau, J.; Tannock, I. F.; Romel, L.; Gore, M.; Ironside, J.; MacDougall, R. H.; Heise, C. et al. A controlled trial of intratumoral ONYX-015, a selectivelyreplicating adenovirus, in combination with cisplatin and 5fluorouracil in patients with recurrent head and neck cancer. Nat. Med. 2000, 6, 879-885.

[103] Jovanović, B.; Mayer, I. A.; Mayer, E. L.; Abramson, V. G.; Bardia, A.; Sanders, M. E.; Kuba, M. G.; Estrada, M. V.; Beeler, J. S.; Shaver, T. M. et al. A randomized phase II neoadjuvant study of cisplatin, paclitaxel with or without everolimus in patients with stage II/III triple-negative breast cancer (TNBC): Responses and long-term outcome correlated with increased frequency of DNA damage response gene mutations, TNBC subtype, AR status, and Ki67. Clin. Cancer Res. 2017, 23, 4035-4045.

[104] Matuszewska, K.; Santry, L. A.; van Vloten, J. P.; AuYeung, A. W. K.; Major, P. P.; Lawler, J.; Wootton, S. K.; Bridle, B. W.; Petrik, J. Combining vascular normalization with an oncolytic virus enhances immunotherapy in a preclinical model of advanced-stage ovarian cancer. Clin. Cancer Res. 2019, 25, 1624-1638.

[105] Saha, D.; Wakimoto, H.; Peters, C. W.; Antoszczyk, S. J.; Rabkin, S. D.; Martuza, R. L. Combinatorial effects of VEGFR kinase inhibitor axitinib and oncolytic virotherapy in mouse and human glioblastoma stem-like cell models. Clin. Cancer Res. 2018, 24, 3409-3422.

[106] Park, A. K.; Fong, Y.; Kim, S. I.; Yang, J.; Murad, J. P.; Lu, J. M.; Jeang, B.; Chang, W. C.; Chen, N. G.; Thomas, S. H. et al. Effective combination immunotherapy using oncolytic viruses to deliver CAR targets to solid tumors. Sci. Transl. Med. 2020, 12, eaaz1863.

[107] Aalipour, A.; Le Boeuf, F.; Tang, M.; Murty, S.; Simonetta, F.; Lozano, A. X.; Shaffer, T. M.; Bell, J. C.; Gambhir, S. S. Viral delivery of CAR targets to solid tumors enables effective cell therapy. Mol. Ther. Oncolytics 2020, 17, 232-240.

[108] Nishio, N.; Diaconu, I.; Liu, H.; Cerullo, V.; Caruana, I.; Hoyos, V.; Bouchier-Hayes, L.; Savoldo, B.; Dotti, G. Armed oncolytic virus enhances immune functions of chimeric antigen receptormodified T cells in solid tumors. Cancer Res. 2014, 74, 5195-5205.

[109] Blake, Z.; Marks, D. K.; Gartrell, R. D.; Hart, T.; Horton, P.; Cheng, 
S. K.; Taback, B.; Horst, B. A.; Saenger, Y. M. Complete intracranial response to talimogene laherparepvec (T-Vec), pembrolizumab and whole brain radiotherapy in a patient with melanoma brain metastases refractory to dual checkpointinhibition. J. Immunother. Cancer 2018, 6, 25.

[110] Kieran, M. W.; Goumnerova, L.; Manley, P.; Chi, S. N.; Marcus, K. J.; Manzanera, A. G.; Polanco, M. L. S.; Guzik, B. W.; AguilarCordova, E.; Diaz-Montero, C. M. et al. Phase I study of genemediated cytotoxic immunotherapy with Adv-tk as adjuvant to surgery and radiation for pediatric malignant glioma and recurrent ependymoma. Neuro Oncol. 2019, 21, 537-546.

[111] Mao, L. J.; Kan, Y.; Li, B. H.; Ma, S.; Liu, Y. R.; Yang, D. L.; Yang, C. H. Combination therapy of prostate cancer by oncolytic adenovirus harboring interleukin 24 and ionizing radiation. Front. Oncol. 2020, 10, 421.

[112] Roulstone, V.; Pedersen, M.; Kyula, J.; Mansfield, D.; Khan, A. A.; McEntee, G.; Wilkinson, M.; Karapanagiotou, E.; Coffey, M.; Marais, R. et al. BRAF- and MEK-targeted small molecule inhibitors exert enhanced antimelanoma effects in combination with oncolytic reovirus through ER stress. Mol. Ther. 2015, 23, 931-942.

[113] Kuryk, L.; Møller, A. S. W.; Garofalo, M.; Cerullo, V.; Pesonen, S.; Alemany, R.; Jaderberg, M. Antitumor-specific T-cell responses induced by oncolytic adenovirus ONCOS-102 (Adv5/3-D24-GMCSF) in peritoneal mesothelioma mouse model. J. Med. Virol. 2018, 90, 1669-1673.

[114] Zhang, F.; Le, T.; Wu, X.; Wang, H.; Zhang, T.; Meng, Y. F.; Wei, B. J.; Soriano, S. S.; Willis, P.; Kolokythas, O. et al. Intrabiliary RF heat-enhanced local chemotherapy of a cholangiocarcinoma cell line: Monitoring with dual-modality imaging-preclinical study. Radiology 2014, 270, 400-408.

[115] Bourgeois-Daigneault, M. C.; Roy, D. G.; Aitken, A. S.; El Sayes, N.; Martin, N. T.; Varette, O.; Falls, T.; St-Germain, L. E.; Pelin, A.; Lichty, B. D. et al. Neoadjuvant oncolytic virotherapy before surgery sensitizes triple-negative breast cancer to immune checkpoint therapy. Sci. Transl. Med. 2018, 10, eaao1641.

[116] Song, J. J.; Zhang, F.; Ji, J. S.; Chen, M. J.; Li, Q.; Weng, Q. Y.; Gu, S. N.; Kogut, M. J.; Yang, X. M. Orthotopic hepatocellular carcinoma: Molecular imaging-monitored intratumoral hyperthermia-enhanced direct oncolytic virotherapy. Int. J. Hyperthermia 2019, 36, 343-349.
[117] Ngwa, V. M.; Edwards, D. N.; Philip, M.; Chen, J. Microenvironmental metabolism regulates antitumor immunity. Cancer Res. 2019, 79, 4003-4008.

[118] Liu, Z. Q.; Ravindranathan, R.; Kalinski, P.; Guo, Z. S.; Bartlett, D. L. Rational combination of oncolytic vaccinia virus and PD-L1 blockade works synergistically to enhance therapeutic efficacy. Nat. Commun. 2017, 8, 14754.

[119] Topalian, S. L.; Drake, C. G.; Pardoll, D. M. Immune checkpoint blockade: A common denominator approach to cancer therapy. Cancer Cell 2015, 27, 450-461.

[120] Chen, D. S.; Mellman, I. Elements of cancer immunity and the cancer-immune set point. Nature 2017, 541, 321-330.

[121] Lichty, B. D.; Breitbach, C. J.; Stojdl, D. F.; Bell, J. C. Going viral with cancer immunotherapy. Nat. Rev. Cancer 2014, 14, 559-567.

[122] June, C. H.; Sadelain, M. Chimeric antigen receptor therapy. N. Engl. J. Med. 2018, 379, 64-73.

[123] O'Cathail, S. M.; Pokrovska, T. D.; Maughan, T. S.; Fisher, K. D.; Seymour, L. W.; Hawkins, M. A. Combining oncolytic adenovirus with radiation-A paradigm for the future of radiosensitization. Front. Oncol. 2017, 7, 153.

[124] Golden, E. B.; Frances, D.; Pellicciotta, I.; Demaria, S.; BarcellosHoff, M. H.; Formenti, S. C. Radiation fosters dose-dependent and chemotherapy-induced immunogenic cell death. Oncoimmunology 2014, 3, e28518.

[125] Ottolino-Perry, K.; Diallo, J. S.; Lichty, B. D.; Bell, J. C.; McCart, J. A. Intelligent design: Combination therapy with oncolytic viruses. Mol. Ther. 2010, 18, 251-263.

[126] Udayakumar, T. S.; Betancourt, D. M.; Ahmad, A.; Tao, W. S.; Totiger, T. M.; Patel, M.; Marples, B.; Barber, G.; Pollack, A. Radiation attenuates prostate tumor antiviral responses to vesicular stomatitis virus containing IFN $\beta$, resulting in pronounced antitumor systemic immune responses. Mol. Cancer Res. 2020, 18, 1232-1243.

[127] Vijayakumar, G.; Palese, P.; Goff, P. H. Oncolytic Newcastle disease virus expressing a checkpoint inhibitor as a radioenhancing agent for murine melanoma. EBioMedicine 2019, 49, 96-105.

[128] Zhang, H. J.; Wang, F.; Mao, C. J.; Zhang, Z. C.; Fu, S. J.; Lu, J. Z.; Zhai, Z. X.; Li, R. J.; Li, S. W.; Rodriguez, R. et al. Effect of combined treatment of radiation and tissue-specific recombinant oncolytic adenovirus on bladder cancer cells. Int. J. Radiat. Biol. 2017, 93, 174-183. 